\title{
Low-energy spectrum of Toeplitz operators: the case of wells
}

\author{
Alix Deleporte* \\ Université de Strasbourg, CNRS, IRMA UMR 7501, F-67000 \\ Strasbourg, France
}

July 7, 2018

\begin{abstract}
In the 1980s, Helffer and Sjöstrand examined in a series of articles the concentration of the ground state of a Schrödinger operator in the semiclassical limit. In a similar spirit, and using the asymptotics for the Szegö kernel, we show a theorem about the localization properties of the ground state of a Toeplitz operator, when the minimal set of the symbol is a finite set of non-degenerate critical points. Under the same condition on the symbol, for any integer $K$ we describe the first $K$ eigenvalues of the operator.
\end{abstract}

\section{Contents}

1 Introduction

1.1 Motivations .....................

1.2 Kähler quantization . . . . . . . . . . . . . . .

1.3 Main results . . . . . . . . . . . . . . . . . .

1.4 Methods - semiclassical properties of Kähler quantization .

1.5 Outline ....................... 9

2 The Szegő projector 9

2.1 Bargmann spaces . . . . . . . . . . . . . . . . . . 9 9

2.2 Semiclassical asymptotics . . . . . . . . . . . . 11

2.3 Universality . . . . . . . . . . . . . . . . . . . . . . . 14

*deleporte@math.unistra.fr

This work was supported by grant ANR-13-BS01-0007-01 
3 Toeplitz operators 15

3.1 Calculus of Toeplitz operators . . . . . . . . . . . 15

3.2 A general localization result . . . . . . . . . . . 16

3.3 Quadratic symbols on the Bargmann spaces ........ 18

4 The first eigenvalue 21

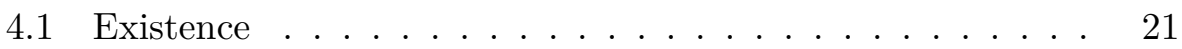

4.2 Positivity . . . . . . . . . . . . . . . . . . . . . . . . 29

4.3 Uniqueness and spectral gap . . . . . . . . . . . . . . . . . . . 30

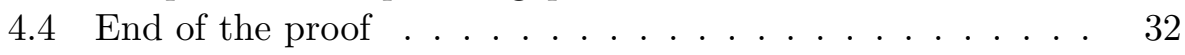

5 Eigenvalues in a scaled window 33

5.1 Approximate eigenvectors . . . . . . . . . . . 33

5.2 Uniqueness . . . . . . . . . . . . . . . . . . . . . . 34

6 Acknowledgements 35

\section{Introduction}

\subsection{Motivations}

In classical mechanics, the minimum of the energy, when it exists, is a critical value, and any point in phase space achieving this minimum corresponds to a stationary trajectory. In quantum mechanics, the situation is quite different. A quantum state cannot be arbitrarily localized in phase space and occupies at least some small amount of space, because of the uncertainty principle. Nevertheless, due to the correspondence principle, one expects the quantum states of minimal energy to concentrate, in some way, near the minimal set of the Hamiltonian, when the effective Planck constant is very small.

In a series of articles [17, 18, 19, 20, 21], Helffer and Sjöstrand considered the Schrödinger operator $P(\hbar)=-\hbar^{2} \Delta+V$, acting on $L^{2}\left(\mathbb{R}^{n}\right)$, where $V$ is a smooth function. If $V$ is smooth, bounded from below and coercive, then the infimum of the spectrum of $P(\hbar)$ is a simple eigenvalue. Helffer and Sjöstrand then studied the concentration properties of associated unit eigenvectors, named ground states, in the semiclassical limit $\hbar \rightarrow 0$. It is well known that the ground state is $O\left(\hbar^{\infty}\right)$ outside any fixed neighbourhood of $\left\{x \in \mathbb{R}^{n}, V(x)=\min (V)\right\}$. If there is only one such $x$, then the ground state concentrates only on $x$. But if there are several minima, it is not clear a priori whether the ground state is evenly distributed on them or not. 
In the first articles [17, 18, 19], the potential $V$ is supposed to reach its minimum only on a finite set of non-degenerate critical points, named "wells". It is proven that only some of the wells are selected by the ground state, that is, the sequence of eigenvectors is $O\left(\hbar^{\infty}\right)$ outside any fixed neighbourhood of this subset of wells. The selected wells are the flattest ones, in a sense that we will make clear later on. Sharper estimates lead to a control, outside of the wells, of the form $\exp \left(-C \hbar^{-1}\right)$, where $C$ is expressed in terms of the Agmon distance to the selected wells. Moreover, when the potential $V$ has two symmetric wells, the ground state "tunnels" between these wells, so that there exists another eigenvalue which is exponentially close to the minimal one.

In the two last papers [20, 21], the potential $V$ is supposed to reach its minimal value on a submanifold of $\mathbb{R}^{n}$. Again, it is easy to prove that the ground state concentrates on this submanifold. From this fact, a formal calculus leads to the study of a Schrödinger operator, on the submanifold, with an effective potential that depends on the 2-jet behaviour of $V$ near the submanifold. The authors treated the case of an effective potential with one non-degenerate minimum, which they call the miniwell condition. In this case, the ground state concentrates only at the miniwell. On the contrary, when the minimal submanifold corresponds to a symmetry of $V$, the ground state is spread out on the submanifold.

This is an instance of what is called quantum selection: not all points in phase space where the classical energy is minimal are equivalent in quantum mechanics. When there is a finite set of minimal points, only some of them are selected by the ground state. Similarly, when the minimal set is a submanifold, the ground state may select only one point (or not). The series of articles [17, 18, 19], and also [34, 35] were adapted to more general pseudodifferential operators [16, 30, 38]. In the physics literature, these effects are believed to appear in other settings; for example, the miniwell condition was used in [14], without mathematical justification, to study quantum selection effects for the Heisenberg model on spin systems, when the classical phase space is a product of 2 -spheres. However, the arguments used by Helffer and Sjöstrand depend strongly on the fact that they deal with Schrödinger operators, when the phase space is $T^{*} \mathbb{R}^{n}$. Thus, it is a priori not clear to which extent the quantum selection can be generalised to a quantization of compact phase spaces.

We propose to study the Kähler quantization, which associates to a symbol on a phase space a Toeplitz operator. In the particular case of the coordinate functions on $\mathbb{S}^{2}=\mathbb{C P}^{1}$, the Toeplitz operators are the spin operators [5], so that our approach contains the physical case of spin sys- 
tems. In this article we prove (Theorem A) that quantum selection occurs in the case of wells, with $O\left(\hbar^{\infty}\right)$ remainder, extending some of the results of [17, 18, 19]. The case of miniwells is in preparation. Exponential estimates will be the object of a separate investigation.

\subsection{Kähler quantization}

When a compact symplectic manifold is endowed with a Kähler structure, there is a natural way to define a quantization scheme, which is compatible with abstract geometric quantization [26, 36].

Definition 1.1. A Kähler manifold is a complex manifold $M$ where the tangent space at each point is endowed with a hermitian metric, i.e. an inner product, whose imaginary part is a closed 2-form on $M$.

In particular, a Kähler manifold has both a symplectic and a Riemannian structure, which are respectively the imaginary part and the real part of the inner product.

Let $L$ be a holomorphic complex line bundle over a Kähler manifold $M$, and $h$ denote a hermitian metric on $L$. Let $\omega$ denote the imaginary part of the hermitian metric. There exists a unique connection (the Chern connection) compatible with $h$ and the complex structure. We wish to consider a prequantum bundle $(L, h)$, such that the curvature of the Chern connection is $-i \omega$. This is always locally possible, but the global existence of a prequantum bundle is equivalent to the fact that $\omega / 2 \pi$ has integral cohomology class (see for instance [40, pp 158-162, or Prop. 2.1.1 of [26]). From now on we suppose that $\omega / 2 \pi$ has integral cohomology class, and we let $(L, h)$ be a prequantum bundle.

There are two equivalent formulations for the basic objects of Kähler quantization, one dealing with holomorphic sections of powers of line bundles [9, 10, 27, the other using equivariant functions on a circle bundle, the Grauert tube [7, 41, 33]. In this article we use the circle bundle approach.

Let $L^{*}$ denote the dual bundle of $L$, with $h^{*}$ the dual metric. It is itself a Riemannian manifold. Define

$$
\begin{aligned}
& D=\left\{(m, v) \in L^{*}, h^{*}(v)<1\right\} \\
& X=\partial D=\left\{(m, v) \in L^{*}, h^{*}(v)=1\right\} .
\end{aligned}
$$

Then $X$ is a circle bundle on $M$, with a $\mathbb{S}^{1}$-action on the fibres $r_{\theta}$ : $(m, v) \mapsto\left(m, e^{i \theta} v\right)$. We will also denote by $\pi$ the projection from $X$ onto $M$. As $X$ is a submanifold of a Riemannian manifold, it inherits a volume form. Since $(L, h)$ is a prequantum bundle, $D$ is pseudoconvex, and the 
volume form on $X$ coincides with the Levi form. The scalar product on $L^{2}(X)$ is related to the one on $L^{2}(M)$ via the $\mathbb{S}^{1}$ action. Indeed, if $s_{0}$ denotes any smooth section of $X$, one has, for any $u, v \in L^{2}(X)$ :

$$
\langle u, v\rangle=\iint_{\mathbb{S}^{1} \times M} \bar{u}\left(r_{\theta} s_{0}(m)\right) v\left(r_{\theta} s_{0}(m)\right) \mathrm{d} \theta \mathrm{d} m .
$$

Let us consider the Hardy space, defined as follows.

Definition 1.2. The Hardy space on $X$, denoted by $H(X)$, is the closed subspace of $L^{2}(X)$ consisting of functions which are boundary values of holomorphic functions inside $D$. The orthogonal projector from $L^{2}(X)$ onto $H(X)$ is denoted by $S$, the Szegö projector.

Using the $\mathbb{S}^{1}$ action, the space $H(X)$ can be further decomposed. For $N \in \mathbb{N}$, an element $f$ of $H$ is said to be $N$-equivariant when, for each $x \in X$ and $\theta \in \mathbb{S}^{1}$, there holds $f\left(r_{\theta} x\right)=e^{i N \theta} f(x)$. The space of $N$-equivariant functions is denoted by $H_{N}(X)$; then $H(X)$ is the orthogonal sum of the different spaces $H_{N}(X)$ for $N \geq 0$. We will call $S_{N}$ the orthogonal projection on $H_{N}(X)$. Then the Schwartz kernel of $S_{N}$ is itself $N$-equivariant, that is:

$$
S_{N}\left(r_{\theta} x, r_{\phi} y\right)=e^{i N(\theta-\phi)} S_{N}(x, y) .
$$

For every $N$, the space $H_{N}(X)$ is finite-dimensional, the dimension growing with $N$. To see this, note that the trace of $S_{N}$ is finite as a consequence of Proposition 2.3. It also comes from the fact that $H_{N}(X)$ can also be formulated as a space of holomorphic sections of an ample line bundle over the compact manifold $M$.

Now we define the Kähler quantization, which associates to any smooth function $f$ on $M$ a sequence of operators $\left(T_{N}(f)\right)_{N \in \mathbb{N}}$ :

Definition 1.3 (Toeplitz operators). Recall $\pi: X \rightarrow M$ is the natural projection. If $f \in C^{\infty}(M)$ is a smooth function, one defines the Toeplitz operator with symbol $f$ as the sequence of operators $T_{N}(f): u \mapsto S_{N}\left(\pi^{*} f u\right)$ from $H_{N}(X)$ to itself.

In this article we are interested in the asymptotics, as $N \rightarrow+\infty$, of Toeplitz operators and their eigenvectors. Alternative conventions exist for the quantization (associating an operator to a symbol), though they define the same class of operators. The convention of Definition 1.3 is sometimes called contravariant [3, 10]. The reason for this choice is that we rely crucially on the positivity condition: if $f$ is real and nonnegative, then $T_{N}(f)$ is nonnegative. 
For any $N$, the operator $T_{N}(f)$ acts on a finite-dimensional space, moreover for real-valued $f$ this operator is obviously self-adjoint. Thus, the spectrum of $T_{N}(f)$ consists only of a finite number of eigenvalues, each of which having a finite multiplicity. We will call the "lowest eigenvalue" the minimum of the spectrum of a Toeplitz operator.

We slightly extend the definition of Toeplitz operators in order to deal with the Kähler manifold $M=\mathbb{C}^{n}$, which is not compact. This does not affect the definitions of $H_{N}(M, L)$ and $S_{N}$, except that the space $H_{N}\left(\mathbb{C}^{n}, L\right)$ has infinite dimension in this case. If $f \in C^{\infty}\left(\mathbb{C}^{n}\right)$, one can define the Toeplitz operator $T_{N}^{\text {flat }}(f)$ as an unbounded operator, and it is an essentially self-adjoint operator with compact resolvent, at least when the symbol is a positive quadratic form (see section 3.3).

\subsection{Main results}

In this article, we adapt the results from [17] to the setting of Kähler quantization. In particular, we are only interested in the following situation:

Definition 1.4. A function $h \in C^{\infty}(M)$ is said to satisfy the wells condition when the following is true:

- $\min (h)=0$

- Every critical point at which $h$ vanishes is non-degenerate.

Observe that, by definition, Morse functions whose minimum is zero satisfy the wells condition, as does the square modulus of a generic holomorphic section of $L^{\otimes N}$ for $N$ large. Note that a function that satisfies the wells condition has a finite cancellation set.

We need the following definition to state our main theorems:

Definition 1.5. Let $Z$ be a subset of $M$, and let

$$
V_{\delta}(N)=\left\{(m, v) \in X, \operatorname{dist}(m, Z)>N^{-\delta}\right\} .
$$

A sequence $\left(u_{N}\right)_{N \in \mathbb{N}}$ of norm 1 functions in $L^{2}(M, L)$ is said to concentrate on $Z$ when, for every $\delta \in\left[0, \frac{1}{2}\right)$, one has

$$
\left\|u_{N} 1_{V_{\delta}(N)}\right\|_{L^{2}(X)}=O\left(N^{-\infty}\right) .
$$

Note that concentration, in the sense of the definition above, implies microsupporting in the sense of Charles [10], that is, for any open set $V$ at positive distance from $Z$, as $N \rightarrow+\infty$, one has $\left\|u_{N} 1_{V}\right\|_{L^{2}}=O\left(N^{-\infty}\right)$. The 
microsupport is contained in the concentration set, while the concentration set is included in any open neighbourhood of the microsupport.

In Subsection 2.2, we consider convenient local maps of "normal coordinates" around any point $P \in M$, which preserve infinitesimally the Kähler structure. If a non-negative function $h$ vanishes with positive Hessian at $P \in M$, the 2-jet of $h$ at $P$ reads in these coordinates as a positive quadratic form $q(P)$ on $\mathbb{C}^{n}$. The first eigenvalue $\mu$ of the Toeplitz operator $T_{1}^{\text {flat }}(q(P))$ (which we call model quadratic operator) does not depend on the choice of normal coordinates. We define this value to be $\mu(P)$.

Let now $h$ be a smooth function on $M$ that satisfies the wells condition of Definition 1.4.

Theorem A. For every $N \in \mathbb{N}$, let $\lambda_{N}$ be the first eigenvalue of the operator $T_{N}(h)$, and $u_{N}$ an associated normalized eigenfunction. Then the sequence $\left(u_{N}\right)_{N \in \mathbb{N}}$ concentrates on the vanishing points of $h$ on which $\mu$ is minimal.

If there is only one such point $P_{0}$, then there is a real sequence $\left(a_{k}\right)_{k \geq 0}$ with $a_{0}=\mu\left(P_{0}\right)$ such that, for each $K$, one has

$$
\lambda_{N}=N^{-1} \sum_{k=0}^{K} N^{-k} a_{k}+O\left(N^{-K-2}\right) .
$$

Moreover, $\lambda_{N}$ is simple, and there exists $C>0$ such that $\lambda_{N}$ is the only eigenvalue of $T_{N}$ in the interval $\left[0, N^{-1}\left(\mu\left(P_{0}\right)+C\right)\right]$.

Theorem B. Let $C>0$. There is a bounded number of eigenvalues (counted with multiplicity) of $T_{N}(h)$ in the interval $\left[0, C N^{-1}\right]$. More precisely, for $C^{\prime}>C$, let $K$ and $\left(b_{k}\right)_{1 \leq k \leq K}$ be such that

$$
\left\{b_{k}, k \leq K\right\}=\bigcup_{\substack{P \in M \\ h(P)=0}} \operatorname{Sp}\left(T_{1}^{\text {flat }}(q(P))\right) \cap\left[0, C^{\prime}\right]
$$

with multiplicity. Then one can find $c>0$ and a list of real numbers $\left(c_{k}\right)_{1 \leq k \leq K}$ such that, for each $k$, one of the eigenvalues of $T_{N}(h)$ lies in the interval

$$
\left[N^{-1} b_{k}+N^{-3 / 2} c_{k}-c N^{-2}, N^{-1} b_{k}+N^{-3 / 2} c_{k}+c N^{-2}\right] .
$$

Moreover, there are at most $K$ eigenvalues of $T_{N}(h)$ in $\left[0, C N^{-1}\right]$ and each of them belongs to one of the intervals above.

Among the smooth functions satisfying the wells condition, there is a dense open subset of "non-resonant" symbols such that, for every $k \geq 0$, the $k$-th eigenvalue of the associated operator has an asymptotic expansion in powers of $N^{-1 / 2}$. 
The case of "miniwells", a transposition of [20], will be treated in future work. Under analyticity conditions, we also hope to state results on exponential decay in the forbidden region, as in [19]. In the one-dimensional case, a full asymptotic expansion for the first eigenvalues of $T_{N}(h)$ was given in [27, with a fixed domain of validity $\left[0, E_{0}\right]$.

\subsection{Methods - semiclassical properties of Kähler quantiza- tion}

If $M$ is compact or $\mathbb{C}^{n}$, the Kähler quantization has many similarities with the Weyl quantization on cotangent bundles. One can indeed find a star product on the space of formal series $C^{\infty}(M)[[\eta]]$ that coincides with the composition of Toeplitz operators when $\eta=N^{-1}$ (see [32] for a short proof), that is, such that

$$
T_{N}(f \star g)=T_{N}(f) T_{N}(g)+O\left(N^{-\infty}\right) .
$$

Thus, the limit $N \rightarrow+\infty$ for Toeplitz operators can be thought of as a semiclassical limit, with semiclassical parameter $N^{-1} \rightarrow 0$. Unless otherwise stated, we will state results under this limit.

It has been known since at least [10] that there is a microlocal equivalence between the semiclassical calculus of Toeplitz operators and that of Weyl quantization. Such a correspondence was already given in the homogeneous setting (without a semiclassical parameter) in [7].

Thus, a possible approach to the spectral study of Toeplitz operators (such as this one, which focuses on low-lying eigenvalues) would be a conjugation by a Fourier Integral Operator to an operator known by previous work. This could be a pseudodifferential operator, in the spirit of [17], or a Toeplitz operator with a simpler symbol, cf [37, 31]. However, each of these approaches require a priori results on the concentration of eigenvectors.

We will use a direct approach in this paper. Indeed, our future work (in preparation) will focus on the case when the minimal set of the symbol is a submanifold, where a priori concentration is not known, so it is unclear whether the previous approaches are sufficient. Moreover, the main theorems in this paper depend on subprincipal effects, and the criterion for quantum selection would be less natural if we should keep track of it through a Fourier Integral Operator. Finally, we believe that Proposition 3.1 is of independent interest. It can easily be generalized into a result on the microsupport of low-energy states for any smooth symbol, and it does not depend on estimates on the asymptotics of the Szegö kernel but only on the nature and symbolic calculus of Toeplitz operators. It could be used as an elementary proof of microsupporting for pseudodifferential operators. 


\subsection{Outline}

We review in Section 2 the definitions and semiclassical properties of the Szegö kernel. Using well-known results about its semiclassical expansion [33, 10, 13, 4], we derive Proposition 2.7, which states that the Szegö kernel on $\mathbb{C}^{n}$ is a local model for any Szegö kernel.

In Section 3, we remind the reader of the symbolic properties of Toeplitz operators [32. The state of the art is such that one can compose Toeplitz operators with classical symbols. We then show, with a new method, a standard result on localization: low-energy eigenvalues concentrate where the symbol is minimal. Finally, we study in detail a particular case of Toeplitz operators, when the base manifold is $\mathbb{C}^{n}$ and the symbol is a positive quadratic form.

Section 4 is devoted to the proof of Theorem A. We build an approximate eigenfunction of the Toeplitz operator and prove that the corresponding eigenvalue is the lowest one. The most important part is Proposition 4.2 for which we use the same method than in [17]. For this, we consider the Hessian of $h$ at a cancellation point, as read in local coordinates; this is a real quadratic form $q$ on $\mathbb{C}^{n}$. Then we compare the Toeplitz operator $T_{N}(h)$ with the Toeplitz operator $T_{N}^{\text {flat }}(q)$, which we call model quadratic operator.

In Section 5, we modify the arguments used in Section 4 to describe, under the same hypotheses on the symbol, the spectrum of a Toeplitz operator in the interval $\left[0, C N^{-1}\right]$ where $C>0$ is arbitrary (Theorem B).

The Appendix is independent from the two main results of the paper. We recover, in the Kähler setting, the off-diagonal estimate for the Szegó kernel of [13, 10, 4, in local coordinates. For this we use the techniques developed in [41, 33], which yield estimates on a shrinking scale, and slightly modify them to recover an estimate on a fixed scale.

\section{The Szegő projector}

\subsection{Bargmann spaces}

As a helpful illustration for the general case (which originates from [1, 2, see also [15], pp. 39-51), we first consider the usual $n$-dimensional complex space $\mathbb{C}^{n}$, with the natural Kähler structure, with $\omega=\sum_{i=1}^{n} \mathrm{~d} z_{i} \wedge \mathrm{d} \bar{z}_{i}$. In this example, the Szegö kernel is explicit.

Because $\mathbb{C}^{n}$ is contractible, the bundle $L$ is isomorphic to $\mathbb{C}^{n+1}$, but the hermitian structure $h$ is not the flat one, for which the associated curvature is zero. Indeed, one can show that $h(m, v)=e^{-|m|^{2}}|v|^{2}$ is the correct choice. 
Here, the spaces $H_{N}\left(\mathbb{C}^{n}, L\right)$ are called the Bargmann spaces and will be denoted by $\mathcal{B}_{N}$. They can be expressed as

$$
\mathcal{B}_{N}=L^{2}\left(\mathbb{C}^{n}\right) \cap\left\{z \mapsto e^{-\frac{N}{2}|z|^{2}} f(z), f \text { holomorphic in } \mathbb{C}^{n}\right\} .
$$

The space $\mathcal{B}_{N}$ is a closed subspace of the Hilbert space $L^{2}\left(\mathbb{C}^{n}\right)$ and inherits its scalar product:

$$
\langle f, g\rangle=\int_{\mathbb{C}^{n}} \bar{f} g .
$$

The functions in $\mathcal{B}_{N}$ are not holomorphic for the standard structure. However, let us introduce the following deformation of $\bar{\partial}$ :

$$
\overline{\mathfrak{d}}_{N}=e^{-\frac{N}{2}|z|^{2}} \bar{\partial} e^{\frac{N}{2}|z|^{2}}=\bar{\partial}+\frac{N}{2} z .
$$

We will further denote by $\overline{\mathfrak{d}}_{N i}$ the $i$-th component of $\overline{\mathfrak{d}}_{N}$. The space $\mathcal{B}_{N}$ is the space of $L^{2}$ functions in the kernel of $\overline{\mathfrak{d}}_{N}$. The adjoint of $\overline{\mathfrak{d}}_{N}$ is $\mathfrak{d}_{N}=e^{-\frac{N}{2}|z|^{2}} \partial e^{\frac{N}{2}|z|^{2}}$. The orthogonal projector on $\mathcal{B}_{N}$ has a Schwartz kernel. Indeed, one Hilbert basis of $\mathcal{B}_{N}$ is the family $\left(e_{\nu}\right)_{\nu \in \mathbb{Z}^{n}}$ with

$$
e_{\nu}(z)=N^{n} \frac{N^{|\nu| / 2} z^{\nu}}{\pi^{n} \sqrt{\nu !}} e^{-\frac{N}{2}|z|^{2}}
$$

Hence the kernel may be expressed as:

$$
\Pi_{N}(x, y)=\frac{N^{n}}{\pi^{n}} \exp \left(-\frac{N}{2}|x|^{2}-\frac{N}{2}|y|^{2}+N x \cdot \bar{y}\right) .
$$

Note that, by definition, $\Pi_{N}$ commutes with $\overline{\mathfrak{d}}_{N}$. Moreover

$$
\left[\Pi_{N}, \bar{z}_{i}\right]=\Pi_{N} \mathfrak{d}_{N i}
$$

The space $\mathcal{B}_{N}$ is isometric to $\mathcal{B}_{1}$ by an isometric dilatation (or scaling) of factor $N^{1 / 2}$ :

$$
\begin{aligned}
\mathcal{B}_{N} & \leftrightarrow \mathcal{B}_{1} \\
f & \mapsto N^{-n} f\left(N^{-1 / 2} \cdot\right) .
\end{aligned}
$$

Moreover, there is a unitary transformation between $\mathcal{B}_{1}$ and $L^{2}\left(\mathbb{R}^{n}\right)$, called the Bargmann transform. The transformation $B_{1}: L^{2}\left(\mathbb{R}^{n}\right) \mapsto \mathcal{B}_{1}$ reads:

$$
B_{1} f(z)=e^{-\frac{1}{2}|z|^{2}} \int \exp \left[-\left(\frac{1}{2} z \cdot z+\frac{1}{2} x \cdot x-\sqrt{2} z \cdot x\right)\right] f(x) \mathrm{d} x .
$$


This transformation conjugates the position operators $z_{i}$ into the position operators $x_{i}$, and the momentum operators $\mathfrak{d}_{1 i}$ into the momentum operators $\frac{1}{i} \frac{\partial}{\partial x_{i}}$.

From $B_{1}$, one can deduce an isometry from $\mathcal{B}_{N}$ to $L^{2}\left(\mathbb{R}^{n}\right)$ by composing the scaling isometry and the Bargmann transform.

One noteworthy subspace of $\mathcal{B}_{1}$ is the dense subset of functions $f \in \mathcal{B}_{1}$ such that $f P \in \mathcal{B}_{1}$ for any polynomial $P \in \mathbb{C}[z]$. This space is denoted by $\mathcal{D}$. Any element of the previously given Hilbert basis belongs to $\mathcal{D}$ and the Bargmann transform is a bijection from $\mathcal{S}\left(\mathbb{R}^{n}\right)$ to $\mathcal{D}$; the preimage of $e_{\nu}$ is the function $x \mapsto C_{\nu} x^{\nu} e^{-|x|^{2} / 2}$, where $C_{\nu}$ is a normalizing factor. Moreover, because of the commutation relations above, the image of $\mathcal{S}\left(\mathbb{C}^{n}\right)$ by the Szegö projector $\Pi_{N}$ is $\mathcal{D}$.

\subsection{Semiclassical asymptotics}

Semiclassical expansions of $S_{N}$ are derived in 41, 33, 28, 9, 44, in different settings. In [41, 33, the Fourier Integral Operator approach is used to prove an asymptotic expansion of $S_{N}$ in a neighbourhood of size $N^{-1 / 2}$ of a point. In [9, 28, 4], one derives asymptotic expansions of $S_{N}$ in a neighbourhood of fixed size of a point.

The Szegö kernel is rapidly decreasing away from the diagonal as $N \rightarrow$ $+\infty$ :

Proposition 2.1 ([10], Corollary 1, or [13], Prop. 4.1 in a more general setting). For every $k \in \mathbb{N}$ and $\epsilon>0$, there exists $C>0$ such that, for every $N \in \mathbb{N}$, for every $x, y \in X$, if

$$
\operatorname{dist}(\pi(x), \pi(y)) \geq \epsilon,
$$

then

$$
\left|S_{N}(x, y)\right| \leq C N^{-k} .
$$

The analysis of the Szegő kernel near the diagonal requires a convenient choice of coordinates. Let $P_{0} \in M$. The real tangent space $T_{P_{0}} M$ carries a Euclidian structure and an almost complex structure coming from the Kähler structure on $M$. We then can (non-uniquely) identify $\mathbb{C}^{n}$ with $T_{P_{0}} M$.

Definition 2.2. Let $U$ be a neighbourhood of 0 in $\mathbb{C}^{n}$ and $V$ be a neighbourhood of $P_{0}$ in $M$. Let $\pi$ denote the projection from $X$ to $M$. Let $\mathbb{R}$ cover $\mathbb{S}^{1}$. The group action $r_{\theta}: \mathbb{S}^{1} \rightarrow X$ lifts to a periodic action from $\mathbb{R}$ to $X$, which we will also call $r_{\theta}$. A smooth diffeomorphism $\rho: U \times \mathbb{R} \rightarrow \pi^{-1}(V)$ is said to be a normal map or map of normal coordinates under the following conditions: 
- $\forall z \in U, \forall \theta \in \mathbb{R}, \rho(z, \theta)=r_{\theta} \rho(z, 0)$;

- Identifying $\mathbb{C}^{n}$ with $T_{P_{0}} M$ as previously, one has:

$$
\forall(z, \theta) \in U \times \mathbb{R}, \pi(\rho(z, \theta))=\exp (z) .
$$

Through this paper we will often read the kernel of $S_{N}$ in normal coordinates. Let $P_{0} \in X$ and $\rho$ a normal map on $X$ such that $\rho(0,0)=P_{0}$. For $z, w \in \mathbb{C}^{n}$ small enough and $N \in \mathbb{N}$, let

$$
S_{N}^{P_{0}}(z, w):=e^{-i N(\theta-\phi)} S_{N}(\rho(z, \theta), \rho(w, \phi)),
$$

which does not depend on $\theta$ and $\phi$ as $S_{N}$ is $N$-equivariant.

The following proposition states that, as $N \rightarrow+\infty$, in normal coordinates, the Szegö kernel has an asymptotic expansion whose first term is the flat kernel of equation (11):

Proposition 2.3 ([13], theorem 4.18). There exist $C>0, C^{\prime}>0, m \in \mathbb{N}$, $\epsilon>0$ and a sequence of polynomials $\left(b_{j}\right)_{j \geq 1}$, with $b_{j}$ of same parity as $j$, such that, for any $N \in \mathbb{N}, K \geq 0$ and $|z|,|w| \leq \epsilon$, one has:

$$
\begin{aligned}
& \left|S_{N}^{P_{0}}(z, w)-\Pi_{N}(z, w)\left(1+\sum_{j=1}^{K} N^{-j / 2} b_{j}(\sqrt{N} z, \sqrt{N} w)\right)\right| \leq \\
& C N^{n-(K+1) / 2}(1+|\sqrt{N} z|+|\sqrt{N} w|)^{m} e^{-C^{\prime} \sqrt{N}|z-w|}+O\left(N^{-\infty}\right) .
\end{aligned}
$$

Remark 2.4. We will use Proposition 2.3 as a black box, as we do not want to divert the reader into considerations on the asymptotics of $S_{N}$, which are more technical than the rest of this paper.

The scope of [13] is much more general than the case of Kähler manifolds; by specialising to this case, one obtains stronger estimates. Indeed, a result very close to this proposition can be found in [4], and also in [10, Theorem 2. However, these results are stated without local coordinates, hence the link with the Bargmann spaces is not obvious.

For the sake of the argument, we derive in the Appendix a precised formulation of this stronger version, adapting the techniques presented in [33].

Remark 2.5. The Proposition 2.3 gives asymptotics for the kernel of $S_{N}$, read in local coordinates. However, the normal maps of Definition 2.2 do not preserve the volume form, except infinitesimally on the fibre over $P_{0}$. For the associated operators to be preserved, one has to pull-back Schwartz 
kernels as half-forms. We claim that it does not change the structure of the asymptotics.

Indeed, if $\mathrm{dVol}$ is the volume form on $X$ and $\mathrm{d} L e b$ is the Lebesgue form on $\mathbb{C}^{n}$, one has, for any normal map $\rho$ :

$$
\rho^{*}(\mathrm{~d} L e b \otimes \mathrm{d} \theta)=a \mathrm{dVol},
$$

for some function $a$ on the domain of $\rho$ with $a(0)=1$. We want to study the asymptotics of $(z, w) \mapsto S_{N}^{P_{0}}(z, w) \sqrt{a(z) a(w)}$, which is the kernel of the pull-back of $S_{N}$.

The function $(z, w) \mapsto \sqrt{a(z) a(w)}$ is smooth on the domain of $\rho$. We write the Taylor expansion of this function at 0 as:

$$
\sqrt{a(z) a(w)}=1+\sum_{j=1}^{K} a_{j}(z, w)+O\left(|z|^{K+1},|w|^{K+1}\right)
$$

where $a_{j}$ is homogeneous of degree $j$, so that $a_{j}(z, w)=N^{-j / 2} a_{j}(\sqrt{N} z, \sqrt{N} w)$.

We let now $\widetilde{b}_{j}$ be such that

$$
\begin{array}{r}
\left(1+\sum_{j=1}^{K} N^{-j / 2} b_{j}(\sqrt{N} z, \sqrt{N} w)\right)\left(1+\sum_{j=1}^{K} N^{-j / 2} a_{j}(\sqrt{N} z, \sqrt{N} w)\right) \\
=1+\sum_{j=1}^{K} N^{-j / 2} \widetilde{b}_{j}(\sqrt{N} z, \sqrt{N} w)+O\left(N^{-(K+1) / 2}\right) .
\end{array}
$$

Then

$$
\begin{array}{r}
\left|S_{N}^{P_{0}}(z, w) \sqrt{a(z) a(w)}-\Pi_{N}(z, w)\left(1+\sum_{j=1}^{K} N^{-j / 2} \widetilde{b}_{j}(\sqrt{N} z, \sqrt{N} w)\right)\right| \leq \\
C N^{n-(K+1) / 2}(1+|\sqrt{N} z|+|\sqrt{N} w|)^{m} e^{-C^{\prime} \sqrt{N}|z-w|}+O\left(N^{-\infty}\right) .
\end{array}
$$

Hence, the effects of the volume form can be absorbed in the error terms of equation (2), and the Proposition 2.3 also holds when $S_{N}$ is replaced by the corresponding half-form.

Thus, we can use the asymptotics of Proposition 2.3 to study how the operator $S_{N}$ acts. For instance, we are able to refine the Proposition 2.1;

Corollary 2.6. For every $k \in \mathbb{N}$ and $\delta \in[0,1 / 2)$, there exists $C>0$ such that, for every $N \in \mathbb{N}$, for every $x, y \in X$ with $\operatorname{dist}(\pi(x), \pi(y)) \geq N^{-\delta}$, one has:

$$
\left|S_{N}(x, y)\right| \leq C N^{-k}
$$


In particular, if $u \in L^{2}(X)$ is $O\left(N^{-\infty}\right)$ outside the pull-back of a ball of size $N^{-\delta}$, then $S_{N}(u)$ is $O\left(N^{-\infty}\right)$ outside the pull-back of a ball of size $2 N^{-\delta}$.

\subsection{Universality}

In the previously given local expansions of the Szegö kernel (2), the dominant term is the projector on the Bargmann spaces of equation (11). Thus the Bargmann spaces appear to be a universal model for Hardy spaces, at least locally. To make this intuition more precise, we derive a useful proposition.

We can pull-back by a normal map the kernel of the projector $\Pi_{N}$ by the following formula:

$$
\rho^{*} \Pi_{N}(\rho(z, \theta), \rho(w, \phi)):=e^{i N(\theta-\phi)} \Pi_{N}(z, w) .
$$

By convention, $\rho^{*} \Pi_{N}$ is zero outside $\pi^{-1}(V)^{2}$.

Proposition 2.7 (Universality). Let $\epsilon>0$. There exists $\delta \in(0,1 / 2)$, a constant $C>0$ and an integer $N_{0}$ such that, for any $N \geq N_{0}$, for any function $u \in L^{2}(X)$ whose support is contained in the fibres over a ball on $M$ of radius $N^{-\delta}$, one has:

$$
\left\|\left(\rho^{*} \Pi_{N}\right) u-S_{N} u\right\|_{L^{2}(X)} \leq C N^{-1 / 2+\epsilon}\|u\|_{L^{2}(X)} .
$$

Proof. Let again $S_{N}^{P_{0}}:(z, \theta, w, \phi) \mapsto e^{-i N(\theta-\phi)} S_{N}(\rho(z, \theta), \rho(w, \phi))$ denote the kernel $S_{N}$ as read in local coordinates, which does not in fact depend on $(\theta, \phi)$.

Equation (2), for $K=0$, can be formulated as:

$$
S_{N}^{P_{0}}(z, w)=\Pi_{N}(z, w)+R(z, w)+O\left(N^{-\infty}\right),
$$

with

$$
|R(z, w)| \leq C N^{n-1 / 2}(1+|\sqrt{N} z|+|\sqrt{N} w|)^{m} e^{-C^{\prime} \sqrt{N}|z-w|}
$$

for every $z$ and $w$ such that $(z, 0)$ and $(w, 0)$ belong to the domain of $\rho$.

Let $\delta \in(0,1 / 2)$ and $u$ a function contained in the pull-back of a ball of size $N^{-\delta}$.

Let $v=S_{N} u-\left(\rho^{*} \Pi_{N}\right) u$. Because of Corollary 2.6, $v$ is $O\left(N^{-\infty}\right)$ outside $\rho\left(B\left(0,4 N^{-\delta}\right) \times \mathbb{S}^{1}\right)$. Hence, up to a $O\left(N^{-\infty}\right)$ error, it is sufficient to control the kernel of $S_{N}-\rho^{*} \Pi_{N}$ on $\rho\left(B\left(0,4 N^{-\delta}\right) \times \mathbb{S}^{1}\right) \times \rho\left(B\left(0,4 N^{-\delta}\right) \times \mathbb{S}^{1}\right)$, where equation (3) is valid.

It remains to estimate the norm of the operator with kernel $R$, using a standard result of operator theory: 
Lemma 2.8 (Schur test). Let $k \in C^{\infty}(V \times V)$ be a smooth function of two variables in an open subset $V$ of $\mathbb{R}^{d}$. Let $K$ be the associated unbounded operator on $L^{2}(V)$.

Let

$$
\|k\|_{L^{\infty} L^{1}}:=\max \left(\sup _{x \in V}\|k(x, \cdot)\|_{L^{1}(V)}, \sup _{y \in V}\|k(\cdot, y)\|_{L^{1}(V)}\right) .
$$

If $\|k\|_{L^{\infty} L^{1}}$ is finite, then $K$ is a bounded operator. Moreover

$$
\|K\|_{L^{2}(V) \mapsto L^{2}(V)} \leq\|k\|_{L^{\infty} L^{1}} .
$$

Thus, we want to estimate the quantity:

$$
\sup _{|z| \leq 4 N^{-\delta}} \int_{|w| \leq 4 N^{-\delta}} N^{n-1 / 2}(1+|\sqrt{N} z|+|\sqrt{N} w|)^{m} e^{-C^{\prime}|z-w|} .
$$

After a change of variables and up to a multiplicative constant, it remains to estimate:

$$
N^{-1 / 2} \sup _{|z| \leq 4 N^{1 / 2-\delta}} \int_{|u| \leq 4 N^{1 / 2-\delta}}(1+|z|+|u|)^{m} e^{-C|u|} .
$$

This quantity is $O\left(N^{(m-1) \frac{1}{2}-m \delta}\right)$. Thus, for any $\epsilon>0$, there exists $\delta$ such that the above quantity is $O\left(N^{-\frac{1}{2}+\epsilon}\right)$.

By the Schur test, the $L^{2}$ norm of a symmetric kernel operator is controlled by the $L^{\infty} L^{1}$ norm of the kernel. When restricted on $B\left(0,4 N^{-\delta}\right)^{2}$, the kernel of $S_{N}^{P_{0}}-\Pi_{N}$ has a $L^{\infty} L^{1}$ norm of order $N^{-\frac{1}{2}+\epsilon}$, from which we can conclude.

\section{Toeplitz operators}

\subsection{Calculus of Toeplitz operators}

The composition of two Toeplitz operators is a formal series of Toeplitz operators. The theorem 2.2 of [32] states for instance that there exists a formal star-product on $C^{\infty}(M)[[\eta]]$, written as $f \star g=\sum_{j=0}^{+\infty} \eta^{j} C_{j}(f, g)$, that coincides with the Toeplitz operator composition: as $N \rightarrow+\infty$, one has, for every integer $K$, that

$$
T_{N}(f) T_{N}(g)-\sum_{j=0}^{K} N^{-j} T_{N}\left(C_{j}(f, g)\right)=O\left(N^{-K-1}\right) .
$$

The functions $C_{j}$ are bilinear differential operators of degree less than $2 j$, and $C_{0}(f, g)=f g$. An explicit derivation of $C_{j}(f, g)$ is given by the Proposition 6 of [10]. 


\subsection{A general localization result}

Using the $C^{*}$-algebra structure of Toeplitz operators, one can prove a fairly general localization result:

Proposition 3.1. Let $h$ be a smooth nonnegative function on $M$. Let $Z=\{h=0\}$, and suppose that $h$ vanishes exactly at order 2 on $Z$, that is, there exists $c>0$ such that $h \geq c \operatorname{dist}(\cdot, Z)^{2}$.

Let $t>0$, and define

$$
V_{t}:=\{(m, v) \in X, \operatorname{dist}(m, Z)<t\} .
$$

For every $k \in \mathbb{N}$, there exists $C>0$ such that, for every $N \in \mathbb{N}$, for every $t>0$, and for every $u \in H_{N}(X)$ such that $T_{N}(h) u=\lambda u$ for some $\lambda \in \mathbb{R}$, one has

$$
\left\|u 1_{X \backslash V_{t}}\right\|_{L^{2}}^{2} \leq C\left(\frac{\max \left(\lambda, N^{-1}\right)}{t^{2}}\right)^{k}\|u\|_{L^{2}}^{2} .
$$

Remark 3.2. Here $M$ is a Kähler manifold, so dist is the Riemannian distance, but since $M$ is compact, the condition on $h$ does not depend on the chosen Riemannian structure.

Proof. By a trivial induction, the $k$-th star power of a symbol $f$ is of the form

$$
f^{\star k}=f^{k}+\eta C_{1, k}(f, \cdots, f)+\eta^{2} C_{2, k}(f, \cdots, f)+\ldots,
$$

where $C_{i, k}$ is a $k$-multilinear differential operator of order at most $2 i$.

We want to study $C_{i, k}(h, \cdots, h)$ for $i \leq k$. The function $h$ is smooth and nonnegative, hence $\sqrt{h}$ is a Lipschitz function. In other terms, there exists $C$ such that, for every $(x, \xi) \in T M$ with $\|\xi\| \leq 1$, one has $\left|\partial_{\xi} h(x)\right| \leq$ $C \sqrt{h(x)}$. In local coordinates, the function $C_{i, k}(h, \cdots, h)$ is a sum of terms of the form $a \partial^{\nu_{1}} h \partial^{\nu_{2}} h \ldots \partial^{\nu_{k}} h$, where $\sum_{j=1}^{k}\left|\nu_{j}\right| \leq 2 i$ and $a$ is smooth.

- If $\nu_{j}=0$, then $\partial^{\nu_{j}} h=h$.

- If $\left|\nu_{j}\right|=1$, then $\left|\partial^{\nu_{j}} h\right| \leq C \sqrt{h}$.

- If $\left|\nu_{j}\right| \geq 2$, then $\left|\partial^{\nu_{j}} h\right| \leq C$.

Hence $\left|a \partial^{\nu_{1}} h \partial^{\nu_{2}} h \ldots \partial^{\nu_{k}} h\right| \leq C h^{k-\frac{1}{2} \sum_{j} \min \left(2,\left|\nu_{j}\right|\right)}$, moreover $\sum_{j} \min \left(2,\left|\nu_{j}\right|\right) \leq$ $\sum_{j}\left|\nu_{j}\right| \leq 2 i$, from which we can conclude:

$$
\left|C_{i, k}(h, \cdots, h)\right| \leq C h^{k-i}
$$


This means that, for every $k \geq 0$, the function $h^{\star k}$ is of the form:

$$
h^{\star k}=h^{k}+\sum_{i=1}^{k-1} \eta^{i} f_{i, k}+\eta^{-k} g(\eta)
$$

where $g$ is bounded independently on $\eta$ and where, for each $i$ and $k$ there exists $C$ such that $\left|f_{i, k}\right| \leq C h^{k-i}$.

Using this, we can prove by induction on $k$ that there exists $C_{k}$ such that, for every $N$ and for every eigenvector $u$ of $T_{N}(h)$ with eigenvalue $\lambda$, one has

$$
\left|\left\langle u, h^{k} u\right\rangle\right| \leq C_{k} \max \left(\lambda, N^{-1}\right)^{k}\|u\|^{2} .
$$

Indeed, this is clearly true for $k=1$, because $\langle u, h u\rangle=\lambda\|u\|^{2}$.

Let us suppose that, for all $1 \leq i \leq k$, there exists $C$ such that

$$
\left|\left\langle u, h^{k-i} u\right\rangle\right| \leq C \max \left(\lambda, N^{-1}\right)^{k-i}\|u\|^{2} .
$$

Because $u$ is an eigenvector for $T_{N}(h)$, it is an eigenvector for its powers, hence

$$
T_{N}\left(h^{\star k}\right) u=T_{N}(h)^{k} u+O\left(N^{-\infty}\right)=\lambda^{k} u+O\left(N^{-\infty}\right) .
$$

Replacing $h^{\star k}$ by its expansion and using the fact that $h \geq 0$, we find:

$$
\left|\left\langle u, h^{k} u\right\rangle\right| \leq \lambda^{k}\|u\|^{2}+\sum_{i=1}^{k-1} N^{-i}\left\langle u, f_{i, k} u\right\rangle+C N^{-k}\|u\|^{2} .
$$

Here we used the fact that the function $g$ is bounded.

Now recall $\left|f_{i, k}\right| \leq C_{i, k} h^{k-i}$, and the induction hypothesis:

$$
\left|\left\langle u, h^{k-i} u\right\rangle\right| \leq C_{i} \max \left(\lambda, N^{-1}\right)^{k-i}\|u\|^{2}
$$

for every $i>0$. Hence

$$
\left|\left\langle u, h^{k} u\right\rangle\right| \leq C \max \left(\lambda, N^{-1}\right)^{k}\|u\|^{2}+\sum_{i=1}^{k-1} C_{i, k} C_{i} N^{-i} \max \left(\lambda, N^{-1}\right)^{k-i}\|u\|^{2},
$$

hence there exists $C_{k}$ such that $\left|\left\langle u, h^{k} u\right\rangle\right| \leq C_{k} \max \left(\lambda, N^{-1}\right)^{k}\|u\|^{2}$.

Now we can conclude: for every $k$, there exists $C$ such that, for every $t>0$ one has

$$
\forall z \notin V_{t}, h^{k} \geq C t^{2 k} .
$$

Finally, for every $k$ there exists $C$ such that, for every $N \in \mathbb{N}, t>0$ and $u$ an eigenvector of $T_{N}(h)$ with eigenvalue $\lambda$, there holds

$$
\left\|u 1_{X \backslash V_{t}}\right\|_{L^{2}}^{2} \leq C\left(\frac{\max \left(\lambda, N^{-1}\right)}{t^{2}}\right)^{k}\|u\|_{L^{2}}^{2} .
$$


Recalling Definition 1.5, let us specialize the Proposition 3.1 to the case $\lambda=O\left(N^{-1}\right)$ and $t=N^{-\delta}$ for $0<\delta<1 / 2$ :

Corollary 3.3. Let $u=\left(u_{N}\right)_{N \in \mathbb{N}}$ be a sequence of unit eigenvectors of $T_{N}(h)$, with sequence of eigenvalues $\lambda_{N}=O\left(N^{-1}\right)$. If $h$ vanishes at order two on its zero set, then u concentrates on this set.

We can reformulate the Proposition 3.1 in these terms: if $h$ is a positive smooth function on $M$, which vanishes at order two on its zero set, then any sequence of normalized eigenvectors of $T_{N}(h)$ whose eigenvalues are $O\left(N^{-1}\right)$ concentrates on the zero set of $h$.

\section{Remark 3.4.}

- An independent work by Charles and Polterovich, that appears partially in [11], treats the case of an eigenvalue close to a regular value of the symbol, with a result very similar to Proposition 3.1.

- The proof of Proposition 3.1 uses cancellation at order two only when dealing with $V_{t}$. Indeed, a more general result is

$$
\left\|u 1_{X \backslash V_{t}}\right\|_{L^{2}}^{2} \leq C\left(\frac{\max \left(\lambda, N^{-1}\right)}{\max \left(h(x), x \in V_{t}\right)}\right)^{k}\|u\|_{L^{2}}^{2},
$$

which holds for any smooth $h$ and any eigenfunction $u$ of $T_{N}(h)$ with eigenvalue $\lambda$.

\subsection{Quadratic symbols on the Bargmann spaces}

Toeplitz operators can also be defined in the Bargmann spaces setting, but one should be careful about the domain of such operators.

This section is devoted to a full survey of the quadratic case, which is very useful as a model case for the general setting. Let $q$ be a positive definite quadratic form on $\mathbb{C}^{n}$. Let

$$
\mathcal{A}_{N}=\left\{f \in \mathcal{B}_{N}, \sqrt{q(\cdot)} f(\cdot) \in L^{2}\left(\mathbb{C}^{n}\right)\right\} .
$$

Then $\mathcal{A}_{N}$ is a dense subspace which contains the image of $\mathcal{D}$ by the isomorphism between $\mathcal{B}_{1}$ and $\mathcal{B}_{N}$. It is the domain of the positive quadratic form $t_{N}:(u, v) \mapsto \int q u \bar{v}$, and $\mathcal{A}_{N}$ is closed for the norm $\|u\|_{\mathcal{A}_{N}}^{2}=\|u\|_{L^{2}}^{2}+$ $t_{N}(u, u)$. Moreover, the injection

$$
\left(\mathcal{A}_{N},\|\cdot\|_{\mathcal{A}_{N}}\right) \hookrightarrow\left(\mathcal{B}_{N},\|\cdot\|_{L^{2}}\right)
$$


is compact. Using the usual results of spectral theory, the associated operator $T_{N}^{f l a t}(q)$ is positive and has compact resolvent. The spectrum of $T_{N}^{\text {flat }}(q)$ thus consists of a sequence of nonnegative eigenvalues, whose only accumulation point is $+\infty$.

Observe that, since $q$ is even, $T_{N}^{\text {flat }}(q)$ sends even functions to even functions, and odd functions to odd functions. Moreover, $q$ is 2-homogeneous. Recalling that the normalized scaling on $\mathbb{C}^{n}$ by a factor $N^{1 / 2}$ sends $\mathcal{B}_{N}$ into $\mathcal{B}_{1}$, the conjugation by this scaling sends $T_{N}^{\text {flat }}(q)$ to $N^{-1} T_{1}^{\text {flat }}(q)$.

Proposition 3.5. The first eigenvalue $\mu_{N}$ of $T_{N}^{\text {flat }}(q)$ is simple.

Proof. As $q$ is positive a.e, the quadratic form $t_{N}$ is strictly convex, hence the first eigenvalue is simple.

We now compare Toeplitz quantization with Weyl quantization for quadratic symbols. Let $O p_{W}^{N^{-1}}(q)$ denote the Weyl quantization of $q$, as a symbol in $T^{*} \mathbb{R}^{n} \simeq \mathbb{C}^{n}$, with semiclassical parameter $N^{-1}$ :

$$
O p_{W}^{N^{-1}}(q) u(x)=\frac{N^{n}}{(2 \pi)^{n}} \int e^{i N\langle\xi, x-y\rangle} q\left(\xi, \frac{x+y}{2}\right) u(y) \mathrm{d} y \mathrm{~d} \xi .
$$

Recall that $B_{N}$ is the $N$-th Bargmann transform.

Proposition 3.6. $B_{N} T_{N}^{f l a t}(q) B_{N}^{-1}=O p_{W}^{N^{-1}}(q)+N^{-1} \frac{\operatorname{tr}(q)}{2}$.

In particular, the first eigenvalue of $T_{N}^{\text {flat }}(q)$ is positive.

Proof. These computations belong to the folklore on the topic. Nevertheless, for the comfort of the reader, we recover them explicitly.

It is sufficient to consider the $N=1$ case which is conjugated with the general case through the usual scaling: indeed $O p_{W}^{N^{-1}}(q)=N^{-1} O p_{W}^{1}(q)$.

Here we shorten the notations for the momentum operators: on the Bargmann side, we let $\mathfrak{d}_{j}=\partial_{z_{j}}+\frac{1}{2} \overline{z_{j}}$; on the $\mathbb{R}^{n}$ side, we let $\partial_{x_{j}}=\frac{1}{i} \frac{\partial}{\partial x_{j}}$.

Let $j, k$ be two indices in $[|1, n|]$.

If $q: z \mapsto z_{j} z_{k}=\left(x_{j}+i y_{j}\right)\left(x_{k}+i y_{k}\right)$, then $\operatorname{tr}(q)=0$, so the two operators should coincide. $T_{1}^{\text {flat }}(q)$ is the operator of multiplication by $z_{j} z_{k}$. This operator is conjugated via $B_{1}$ to the operator $\left(x_{j}+i \partial_{x_{j}}\right)\left(x_{k}+i \partial_{x_{k}}\right)=$ $x_{j} x_{k}-\partial_{x_{j}} \partial_{x_{k}}+i x_{j} \partial_{x_{k}}+i \partial_{x_{j}} x_{k}$. Moreover, the Weyl quantization of $q$ is the operator

$$
O p_{W}^{1}(q)=x_{j} x_{k}-\partial_{x_{j}} \partial_{x_{k}}+\frac{i}{2}\left(\partial_{x_{k}} x_{j}+x_{j} \partial_{x_{k}}+\partial_{x_{j}} x_{k}+x_{k} \partial_{x_{j}}\right) .
$$

These two operators coincide whether $j=k$ or not. 
The case $q: z \mapsto \overline{z_{j} z_{k}}=\left(x_{j}-i y_{j}\right)\left(x_{k}-i y_{k}\right)$ is the adjoint of the previous one.

If $q: z \mapsto z_{j} \overline{z_{k}}=\left(x_{j}+i y_{j}\right)\left(x_{k}-i y_{k}\right)$, then $\operatorname{tr}(q)=2 \delta_{k}^{j}$. In that case, $T_{1}^{f l a t}(q)=\mathfrak{d}_{k} z_{j}$. This operator is conjugated to $\left(x_{k}-i \partial_{x_{k}}\right)\left(x_{j}+i \partial_{x_{j}}\right)$. The Weyl quantization of $q$ is

$$
O p_{W}^{1}(q)=x_{j} x_{k}+\partial_{x_{j}} \partial_{x_{k}}+\frac{i}{2}\left(-\partial_{x_{k}} x_{j}-x_{j} \partial_{x_{k}}+\partial_{x_{j}} x_{k}+x_{k} \partial_{x_{j}}\right) .
$$

The two operators coincide when $k \neq j$, and when $k=j$ the difference is 1.

From the conjugation, it is clear that the first eigenvalue of $T_{N}^{f l a t}(q)$ is positive, because the Weyl quantization of $q$ is nonnegative (see Remark 3.7) and $\operatorname{tr}(q)>0$.

Because $T_{N}^{\text {flat }}(q)$ is conjugated to $N^{-1} T_{1}^{\text {flat }}(q)$, one has $\mu_{N}=N^{-1} \mu_{1}$, and for some $C>0$,

$$
\operatorname{dist}\left(\mu_{N}, S p\left(T_{N}^{\text {flat }}\right) \backslash\left\{\mu_{N}\right\}\right)=C N^{-1} .
$$

The first eigenvalue $\mu_{1}$ of $T_{1}(q)$ depends on $q$, but is invariant under an unitary change of variables on $\mathbb{C}^{n}$. From now on we will use the notation $\mu(q)$ to denote $\mu_{1}$.

Remark 3.7. The computation of $\mu(q)$ is non-trivial. As explained in [29], Lemma 2.8, or as a direct consequence of the classification in [39], the first eigenvalue of $O p_{W}^{1}(q)$ can be obtained the following way: let $M \in S_{2 n}^{+}(\mathbb{R})$ denote the symmetric matrix associated with $q$ in the canonical coordinates. Let $J$ be the matrix of the symplectic structure:

$$
J=\left(\begin{array}{cc}
0 & -I d \\
I d & 0
\end{array}\right)
$$

Then the matrix $J M$ is skew-symmetric with respect to the scalar product given by $M$. Hence $A=i J M$ can be diagonalized; the eigenvalues of $A$ appear in pairs of opposite values $\lambda$ and $-\lambda$. Then $\mu$ is the sum of the positive diagonal elements of $A$. In particular, this explicit formulation shows that $O p_{W}^{N^{-1}}(q)$ is nonnegative.

We can use Proposition 3.6 to transpose well-known results for the quantization of quadratic symbols to the Bargmann case. Since $\mu(q)$ is simple, the operator $T_{1}^{\text {flat }}(q)-\mu(q)$ has a continuous inverse on the orthogonal set of the associated eigenfunction. This inverse sends $\mathcal{D}$ into itself, because one can build a Hilbert base of $\mathcal{D}$ with eigenfunctions of $T_{1}^{\text {flat }}(q)$. Moreover the eigenfunction associated with $\mu(q)$ is even. 
Remark 3.8. To illustrate the Proposition 3.6, we solve completely the $n=1$ case. Up to a $U(1)$ change of variable, any real quadratic form on $\mathbb{C}$ can be written as $\alpha x^{2}+\beta y^{2}$. The associated Weyl operator is $-\beta \Delta+\alpha x^{2}$, with first eigenvalue $\sqrt{\alpha \beta}$. On the other hand, the first eigenfunction of $\frac{\alpha-\beta}{4}\left(z^{2}+\mathfrak{d}^{2}\right)+\frac{\alpha+\beta}{2} \mathfrak{d} z$ is a squeezed state of the form $z \mapsto e^{-\frac{1}{2}|z|^{2}} e^{\frac{\lambda}{2} z^{2}}$, with $\lambda=\frac{(\sqrt{\alpha}-\sqrt{\beta})^{2}}{\alpha-\beta}($ or $\lambda=0$ in case $\alpha=\beta)$. The associated eigenvalue is then $\frac{(\sqrt{\alpha}+\sqrt{\beta})^{2}}{2}$. The difference is $\frac{\alpha+\beta}{2}$, which is exactly half of the trace of $q$.

Remark 3.9. If instead of $T_{N}(h)$ one would consider $T_{N}\left(h-\frac{\Delta h}{2 N}\right)$, as in [10], then the Toeplitz quantization of a quadratic form would be exactly conjugated to its Weyl quantization: indeed $\operatorname{tr}(q)=\Delta q$. We recover in this particular case the computations in [27].

\section{The first eigenvalue}

This section is devoted to the proof of Theorem A.

Let $P_{0} \in M$, one can find normal coordinates from a neighbourhood of $P_{0}$ to a neighbourhood of 0 in $\mathbb{C}^{n}$. If at $P_{0}$ a non-negative function $h$ vanishes with positive Hessian, the 2-jet of $h$ at $P_{0}$ maps to a positive quadratic form $q$ on $\mathbb{C}^{n}$, up to a $U(n)$ change of variables. Hence, the map associating to $P_{0}$ the first eigenvalue $\mu$ of the model quadratic operator $T_{N}^{\text {flat }}(q)$ is well-defined. From now on, we will also call $\mu$ this map.

The method of proof for Theorem A is then as follows: for each vanishing point $P_{0}$, we construct a sequence of functions which concentrates on $P_{0}$, consisting of almost eigenfunctions of $T_{N}(h)$, and for which the associated sequence of eigenvalues is equivalent to $N^{-1} \mu\left(P_{0}\right)$ as $N \rightarrow+\infty$. We then show a positivity estimate for eigenfunctions concentrating on a single well. The uniqueness and the spectral gap property follow from a similar argument. At every step, we compare $T_{N}(h)$ with the operator on $\mathcal{B}_{N}$ whose symbol is the Hessian of $h$ at the point of interest.

\subsection{Existence}

We let $h$ denote a smooth function satisfying the wells condition. At every cancellation point of $h$, we will find a candidate for the ground state of $T_{N}(h)$. Instead of finding exact eigenfunctions, we search for approximate eigenfunctions. This is motivated by the following lemma: 
Lemma 4.1. Let $T$ be a self-adjoint operator on a Hilbert space, let $\lambda \in \mathbb{R}$, and $u \in D(T)$ with norm 1 .

Then $\operatorname{dist}(\lambda, S p(T)) \leq\|T(u)-\lambda u\|$.

Let $P_{0} \in M$ be a point where $h$ vanishes. Let $\rho$ be a local map of normal coordinates in a neighbourhood of $\pi^{-1}\left(P_{0}\right)$. Let $\Omega_{N}$ be the set of $z \in \mathbb{C}^{n}$ such that $(z / \sqrt{N}, 0)$ belongs to the domain of $\rho$. Recall from equation (2) that, for every $N \in \mathbb{N}$ and every $z, w \in \Omega_{N}$, there holds:

$$
\begin{aligned}
N^{-n} & S_{N}^{P_{0}}\left(\frac{z}{\sqrt{N}}, \frac{w}{\sqrt{N}}\right) \\
& \left.=\Pi_{1}(z, w)\left(1+\sum_{k=1}^{K} N^{-k / 2} b_{k}(z, w)\right)\right)+R_{K}(z, w, N)+O\left(N^{-\infty}\right) .
\end{aligned}
$$

Here the $b_{j}$ 's are polynomials of the same parity as $j$, and there exist $C>0, m>0$ such that, for every $(z, w, N)$ as above:

$$
\left|R_{K}(z, w, N)\right| \leq C N^{-(K+1) / 2} e^{-C^{\prime}|z-w|}\left(1+|z|^{m}+|w|^{m}\right) .
$$

The main proposition is

Proposition 4.2. There exists a sequence $\left(u_{j}\right)_{j \geq 0}$ of elements of $\mathcal{S}\left(\mathbb{C}^{n}\right)$, with $\left\langle u_{0}, u_{k}\right\rangle=\delta_{k}^{0}$, and a sequence $\left(\lambda_{j}\right)_{j \geq 0}$ of real numbers, with $\lambda_{0}=\mu\left(P_{0}\right)$ and $\lambda_{j}=0$ for $j$ odd, such that, for each $K$ and $N$, if $u^{K}(N) \in L^{2}(X)$ and $\lambda^{K}(N) \in \mathbb{R}$ are defined as:

$$
\begin{gathered}
u^{K}(N)(\rho(z, \theta)):=e^{i N \theta} N^{n} \sum_{j=0}^{K} N^{-j / 2} u_{j}(\sqrt{N} z), \\
u^{K}(N) \text { is supported in the image of } \rho, \\
\lambda^{K}(N)=N^{-1} \sum_{j=0}^{K} N^{-j / 2} \lambda_{j},
\end{gathered}
$$

there holds, as $N \rightarrow+\infty$,

$$
\left\|S_{N} h S_{N} u^{K}(N)-\lambda^{K}(N) u^{K}(N)\right\|_{L^{2}(X)}=O\left(N^{-(K+3) / 2}\right) .
$$

Remark 4.3. The functions $u^{K}(N)$ do not lie inside $H_{N}(X)$, because they are identically zero on an open set. Nevertheless, the operator $S_{N} h S_{N}$ on $L^{2}(X)$ decomposes orthogonally into $T_{N}(h)$ on $H_{N}$, and 0 on its orthogonal. Hence a nonzero eigenvalue of $S_{N} h S_{N}$ must correspond to an eigenvalue of $T_{N}(h)$ with same eigenspace. The same holds for almost eigenvalues. 
Introducing $\lambda^{K}$ as a polynomial in $N^{-1 / 2}$ whose odd terms vanish may seem surprising. However, in the proof, we construct $\lambda^{K}$ as a polynomial in $N^{-1 / 2}$, as we do for $u^{K}$. The fact that it is a polynomial in $N^{-1}$ is due to parity properties.

Proof. Let us solve the successive orders of

$$
\left(S_{N} h S_{N}-\lambda^{K}(N)\right) u^{K}(N) \approx 0 .
$$

We write the Taylor expansion of $h$ around $P_{0}$ at order $K$ as

$$
h(x)=q(x)+\sum_{j=3}^{K} r_{j}(x)+E_{K}(x) .
$$

Because of equation (4), the kernel of $S_{N} h S_{N}$, read in the map $\rho$, is:

$$
\begin{aligned}
& N^{-n} e^{i(\phi-\theta)} S_{N} h S_{N}\left(\rho\left(N^{-1 / 2} z, N^{-1} \theta\right), \rho\left(N^{-1 / 2} w, N^{-1} \phi\right)\right) \\
&= N^{-1} \int\left(q(y)+\sum_{k=1}^{K-2} N^{-k / 2} r_{k+2}(y)+N E_{K}\left(N^{-1 / 2} y\right)\right) \\
& \times\left[\Pi_{1}(z, y)\left(1+\sum_{j=1}^{K} N^{-j / 2} b_{j}(z, y)\right)+R_{K}(z, y, N)\right] \\
& \times {\left[\Pi_{1}(y, w)\left(1+\sum_{l=1}^{K} N^{-l / 2} b_{l}(y, w)\right)+R_{K}(y, w, N)\right] \mathrm{d} y } \\
&+O\left(N^{-\infty}\right) .
\end{aligned}
$$

Let us precisely write down the $K=0$ and $K=1$ case.

The dominant order (that is, $N^{-1}$ ) of the right-hand side is simply:

$$
(z, w) \mapsto N^{-1} \int_{\mathbb{C}^{n}} \Pi_{1}(z, y) q(y) \Pi_{1}(y, w) \mathrm{d} y .
$$

It is $N^{-1}$ times the kernel of the Toeplitz operator $Q=T_{1}^{\text {flat }}(q)$ on $B_{1}$ associated to the quadratic symbol $q$, which we studied in Subsection 3.3. Its resolvant is compact, the first eigenvalue $\mu\left(P_{0}\right)$ is simple, and if $u_{0}$ is an associated eigenvector, the operator $Q-\mu\left(P_{0}\right)$ has a continuous inverse on $u_{0}^{\perp}$ which sends $\mathcal{D}$ into itself. Moreover $u_{0}$ is an even function.

This determines $u_{0}$ and $\lambda_{0}=\mu\left(P_{0}\right)$. Here $u_{0} \in \mathcal{D}$, so we can truncate the function $(z, \theta) \mapsto e^{i N \theta} N^{n} u_{0}\left(N^{1 / 2} z\right)$ to a function supported on the 
domain of $\rho$, with only $O\left(N^{-\infty}\right)$ error. The push-forward by $\rho$ of this truncation, extended by zero outside the image of $\rho$, is denoted by $u^{0}(N)$.

Now $u_{0} \in \mathcal{D}$ so $u^{0}$ concentrates on $P_{0}$. The error is thus:

$$
\begin{aligned}
\| S_{N} h S_{N} u^{0}(N) & -N^{-1} \lambda_{0} u^{0}(N) \|_{L^{2}(X)}^{2} \\
& \leq C N^{-2} \int_{\Omega_{N}^{3}} A(z, y, w, N)^{2}\left|u_{0}(w)\right|^{2} \mathrm{~d} y \mathrm{~d} w \mathrm{~d} z+O\left(N^{-\infty}\right),
\end{aligned}
$$

where

$$
\begin{aligned}
A(z, y, w, N)=N\left|E_{2}\left(N^{-1 / 2} y\right) \Pi_{1}(z, y) \Pi_{1}(y, w)\right| & \\
+h(y)\left(\left|R_{0}(z, y, N)\right| \Pi_{1}(y, w)+\right. & \left|R_{0}(y, w, N)\right| \Pi_{1}(z, y) \\
& \left.+\left|R_{0}(z, y, N) R_{0}(y, w, N)\right|\right) .
\end{aligned}
$$

Here, $E_{2}$ is a Taylor remainder of order 3 on a compact set, so

$$
\left|N E_{2}\left(N^{-1 / 2} y\right)\right| \leq C|y|^{3} N^{-1 / 2} .
$$

Moreover, recall that, on $\Omega_{N}^{2}$, one has

$$
\left|R_{0}(z, y, N)\right| \leq C N^{-1 / 2} e^{-C^{\prime}|z-y|}\left(1+|z|^{m}+|y|^{m}\right) .
$$

Hence, on $\Omega_{N}^{3}$, there holds:

$$
|A(z, y, w, N)| \leq C N^{-1 / 2} e^{-C^{\prime}|z-y|-C^{\prime}|y-w|}\left(1+|z|^{m}+|y|^{m}+|w|^{m}\right) .
$$

Because $u_{0} \in \mathcal{D}$, one deduces:

$$
\begin{aligned}
& N^{3} \int_{X}\left|S_{N} h S_{N} u^{0}-N^{-1} \lambda_{0} u^{0}\right|^{2} \\
& \leq C \int_{\Omega_{N}^{3}} e^{-2 C^{\prime}|z-y|-2 C^{\prime}|y-w|}\left(1+|z|^{2 m}+|y|^{2 m}+|w|^{2 m}\right)\left|u_{0}(w)\right|^{2} \mathrm{~d} y \mathrm{~d} z \mathrm{~d} w \\
& \quad+O\left(N^{-\infty}\right) \\
& \leq C\left(\int_{\mathbb{C}^{n}}|v|^{2 m} e^{-C^{\prime}|v|} \mathrm{d} v\right)^{2} \int_{\mathbb{C}^{n}}|w|^{2 m}\left|u_{0}(w)\right|^{2} \mathrm{~d} w+O\left(N^{-\infty}\right) \\
& \leq C .
\end{aligned}
$$

This method (estimating an error kernel using polynomial growth and off-diagonal exponential decay) will be used repeatedly again. 
From there we deduce that $u_{0}$ is an approximate eigenvector:

$$
\left\|S_{N} h S_{N} u_{0}(N)-N^{-1} \lambda_{0} u_{0}(N)\right\|_{L^{2}(X)}=O\left(N^{-3 / 2}\right) .
$$

This proves the proposition for the case $K=0$.

The construction of $u_{1}$ and $\lambda_{1}$ is different, moreover there are supplementary error terms. The term of order $N^{-3 / 2}$ in the right-hand side of equation (5) is:

$$
(z, w) \mapsto N^{-3 / 2} \int_{\mathbb{C}^{n}} \Pi_{1}(z, y)\left[r_{3}(y)+q(y)\left(b_{1}(z, y)+b_{1}(y, w)\right)\right] \Pi_{1}(y, w) \mathrm{d} y .
$$

Let $J_{1}$ denote the operator with kernel as above. We are trying to find $u_{1}$ and $\lambda_{1}$ such that

$$
\left(Q-\lambda_{0}\right) u_{1}+J_{1} u_{0}=\lambda_{1} u_{0}
$$

with the supplementary condition that $\left\langle u_{1}, u_{0}\right\rangle=0$ : indeed if $\left(u_{1}, \lambda_{1}\right)$ is a solution of equation (6), then so is $\left(u_{1}+c u_{0}, \lambda_{1}\right)$ for any $c \in \mathbb{C}$. The orthogonality condition makes the solution unique as we will see.

The functions $r_{3}, q$ and $b_{1}$ are polynomials, so $J_{1}(\mathcal{D}) \subset \mathcal{S}\left(\mathbb{C}^{n}\right)$. This ensures that the problem is well-posed. Note that $J_{1}$ does not map $\mathcal{D}$ into holomorphic functions; this is because the normal map $\rho$ does not preserve the holomorphic structure.

Now $r_{3}$ and $b_{1}$ are odd, so $J_{1} u_{0}$ is odd. In particular, $\left\langle u_{0}, J_{1} u_{0}\right\rangle=0$, and because $Q$ is self-adjoint, $\left\langle u_{0},\left(Q-\lambda_{0}\right) u_{1}\right\rangle=0$. From this we deduce that $\lambda_{1}\left\|u_{0}\right\|^{2}=0$, hence $\lambda_{1}=0$.

To find $u_{1}$, we use again the fact that $J_{1} u_{0}$ is orthogonal to $u_{0}$. Since $\lambda_{0}$ is a simple eigenvalue, $Q-\lambda_{0}$ is invertible from $u_{0}^{\perp}$ to itself, and maps $\mathcal{S} \cap u_{0}^{\perp}$ to itself. Hence there exists a unique $u_{1} \in \mathcal{S}$ orthogonal to $u_{0}$, such that $\left(u_{1}, 0\right)$ solves (6). Moreover $u_{1}$ is odd. let

Now we investigate the error terms. With $u^{1}$ and $\lambda^{1}$ as in the statement,

$$
f^{1}(N)=\left(S_{N} h S_{N}-\lambda^{1}(N)\right) u^{1}(N) .
$$

As $u_{0}$ and $u_{1}$ belong to $\mathcal{S}$, the function $u^{1}$ concentrates on $P_{0}$, and so does $f^{1}$. Hence it is sufficient to control $f^{1}$ near $P_{0}$. After a change of 
variables, one has:

$$
\begin{gathered}
N^{-n} e^{-i \theta} f^{1}(N)\left(\rho\left(N^{-1 / 2} z, N^{-1} \theta\right)\right)=N^{-2} J_{1} u_{1}(z) \\
+\int \Pi_{1}(z, y) \Pi_{1}(y, w) E_{3}\left(\frac{y}{\sqrt{N}}\right)\left(1+\frac{b_{1}(z, y)}{\sqrt{N}}\right)\left(1+\frac{b_{1}(y, w)}{\sqrt{N}}\right)\left(u_{0}(w)+\frac{u_{1}(w)}{\sqrt{N}}\right) \mathrm{d} y \mathrm{~d} w \\
+N^{-1} \int R_{1}(z, y, N) \Pi_{1}(y, w)\left(1+\frac{b_{1}(y, w)}{\sqrt{N}}\right)\left(q(y)+\frac{r_{3}(y)}{\sqrt{N}}\right)\left(u_{0}(w)+\frac{u_{1}(w)}{\sqrt{N}}\right) \mathrm{d} y \mathrm{~d} w \\
+N^{-1} \int \Pi_{1}(z, y)\left(1+\frac{b_{1}(z, y)}{\sqrt{N}}\right) R_{1}(y, w, N)\left(q(y)+\frac{r_{3}(y)}{\sqrt{N}}\right)\left(u_{0}(w)+\frac{u_{1}(w)}{\sqrt{N}}\right) \mathrm{d} y \mathrm{~d} w \\
+N^{-1} \int R_{1}(z, y, N) R_{1}(y, w, N)\left(q(y)+\frac{r_{3}(y)}{\sqrt{N}}\right)\left(u_{0}(w)+\frac{u_{1}(w)}{\sqrt{N}}\right) \mathrm{d} y \mathrm{~d} w \\
+O\left(N^{-\infty}\right) .
\end{gathered}
$$

As $u_{1} \in \mathcal{S}$, the first line of the right-hand term is well-defined, and $\left\|N^{-2} J_{1} u_{1}\right\|=O\left(N^{-2}\right)$.

There holds a uniform Taylor estimate on the domain of $\rho$ :

$$
E_{3}(y) \leq C|y|^{4},
$$

so $E_{3}\left(N^{-1 / 2} y\right)$ is bounded by $N^{-2}$ times a function with polynomial growth independent of $N$. In particular, there exist $C, C^{\prime}, m>0$ such that, on $\Omega_{N}^{3}$ :

$$
\begin{aligned}
& \mid E_{3}\left(N^{-1 / 2} y\right) \Pi_{1}(z, y) \Pi_{1}(y, w) \mid \\
& \leq C N^{-2} e^{-C^{\prime}|z-y|-C^{\prime}|y-w|}\left(1+|z|^{m}+|y|^{m}+|w|^{m}\right) .
\end{aligned}
$$

Of course the same type of estimate (with different $C$ and $m$ ) applies if we multiply the left-hand side by $b_{1}(z, y), b_{1}(y, w)$, or both. Hence, following the last part of the $K=0$ case, we can estimate the second line of the expansion of $f^{1}$ as $O_{L^{2}(X)}\left(N^{-2}\right)$.

The three following lines are treated the same way: because $u_{0}$ and $u_{1}$ belong to $\mathcal{S}$, it is sufficient to prove estimates for the error kernels, of the form

$$
|A(z, y, w, N)| \leq N^{-2} C e^{-C^{\prime}|z-y|-C^{\prime}|y-w|}\left(1+|z|^{m}+|y|^{m}+|w|^{m}\right),
$$

which are easily checked.

We construct by induction on $K$ the following terms of the expansion. 
For $j \geq 1$, we let $J_{j}: L^{2}\left(\mathbb{C}^{n}\right) \mapsto L^{2}\left(\mathbb{C}^{n}\right)$ the unbounded and symmetric operator with kernel

$$
J_{j}(x, z)=\int_{\mathbb{C}^{n}} \Pi_{1}(x, y) \Pi_{1}(y, z)\left(\sum_{\substack{k+l+m=j \\ k, m, l \geq 0}} b_{k}(x, y) r_{2+l}(y) b_{m}(y, z)\right) \mathrm{d} y .
$$

Here we use the convention $b_{0}=1$, and $r_{2}=q$. The dense subspace $\mathcal{S}\left(\mathbb{C}^{n}\right)$ is included in the domain of $J_{j}$, moreover $J_{j}(\mathcal{S}) \subset \mathcal{S}$ because all the $b_{j}$ 's and $r_{l}$ 's are polynomials. Moreover $J_{j}$ has the same parity as $j$.

Let $K \in \mathbb{N}$, and suppose we found functions $\left(u_{k}\right)_{k \leq K} \in \mathcal{S}$, orthogonal to $u_{0}$, and of the same parity as $k$, and real numbers $\lambda_{k}$ that vanish when $k$ is odd, and such that, for each $k \leq K$, there holds:

$$
\left(Q-\lambda_{0}\right) u_{k}+\sum_{j=1}^{k} J_{j} u_{k-j}=\lambda_{k} u_{0}+\sum_{j=1}^{k-1} \lambda_{j} u_{k-j}
$$

Let us find $u_{K+1}$, orthogonal to $u_{0}$, and $\lambda_{K+1}$ so that equation (7) also holds for $k=K+1$.

Take the scalar product with $u_{0}$. As $Q$ is symmetric, the left-hand side vanishes, and we get a linear equation in $\lambda_{K+1}$, whose dominant coefficient is $\left\|u_{0}\right\|^{2}=1$. Hence $\lambda_{K+1}$ is uniquely determined. Moreover, if $K+1$ is odd, then $J_{j} u_{K+1-j}$ and $\lambda_{j} u_{K+1-j}$ are odd functions for every $j$, so their scalar products with $u_{0}$ are zero, hence $\lambda_{K+1}=0$.

We now are able to find $u_{K+1}$ because we can invert $Q-\lambda_{0}$ on the orthogonal set of $u_{0}$. Finally, $u_{K+1}$ is of the same parity as $K+1$.

It remains to show that this sequence of functions $u$ corresponds to an approximate eigenvector of $S_{N} h S_{N}$.

Let $K \geq 0$, fixed in what follows. For each $N \in \mathbb{N}$, we can build a function $u^{K}(N)$ on $X$, supported in the image of $\rho$ and such that, for $x$ in the image of $\rho$, one has $u^{K}(N)(\rho(z, \theta))=e^{i N \theta} N^{n} \sum_{k=0}^{K} N^{-k / 2} u_{k}(\sqrt{N} z)$. Note that $u^{K}(N)$ concentrates on $P_{0}$.

Let

$$
\lambda^{K}(N)=N^{-1} \sum_{k=0}^{K} N^{-k / 2} \lambda_{k}
$$

We evaluate $\left(S_{N} h S_{N}-\lambda^{K}(N)\right) u^{K}(N)=: f^{K}(N)$. Consider an open set $V_{1}$, containing $P_{0}$, and compactly included in the image of $\rho$. One has

$$
\left\|f^{K}(N)\right\|_{L^{\infty}\left({ }^{c} V_{1}\right)}=O\left(N^{-\infty}\right)
$$

because $u^{K}(N)$ concentrates on $P_{0}$. 
To compute $f^{K}(N)$ in $V_{1}$, we use the equation (44) at order $K$. A change of variables leads to:

$$
\begin{aligned}
& N^{-n} e^{-i N \theta} f^{K}(N)\left(\rho\left(N^{-1 / 2} z, \theta\right)\right) \\
= & N^{-1} \sum_{k=0}^{K} N^{-\frac{k}{2}}\left[\left(Q-\lambda_{0}\right) u_{k}(z)-\sum_{j=1}^{k} J_{j} u_{k-j}(z)-\lambda_{k} u_{0}(z)-\sum_{j=1}^{k-1} \lambda_{j} u_{k-j}(z)\right] \\
& +N^{-1} \sum_{k=K+1}^{2 K} N^{-\frac{k}{2}}\left[-\sum_{j=k-K}^{K}\left(J_{j}-\lambda_{j}\right) u_{k-j}(z)\right] \\
+ & \sum_{k, j, l=0}^{K} N^{-\frac{k+j+l}{2}} A_{j, l, N} u_{k}(z)+\sum_{k, j=0}^{K} N^{-\frac{k+j}{2}} A_{j, N}^{\prime} u_{k}(z)+\sum_{k=0}^{K} N^{-\frac{k}{2}} A_{N}^{\prime \prime} u_{k}(z) .
\end{aligned}
$$

By construction, the first line of the right-hand term vanishes. The second line is $O\left(N^{-(K+3) / 2}\right)$. There are three error terms in the last line. $A_{j, l, N}$ is the operator with kernel:

$$
A_{j, l, N}(z, w)=\int_{\Omega_{N}} \Pi_{1}(z, y) \Pi_{1}(y, w) b_{j}(z, y) b_{l}(y, w) E_{K}\left(N^{-1 / 2} y\right) \mathrm{d} y .
$$

The function $E_{K}$ is a Taylor remainder at order $K+3$, so there exist constants $C>0, C^{\prime}>0, m>0$ such that, on $\Omega_{N}^{3}$ :

$$
\begin{aligned}
& \quad\left|\Pi_{1}(z, y) \Pi_{1}(y, w) b_{j}(z, y) b_{l}(y, w) E_{K}\left(N^{-1 / 2} y\right)\right| \\
& \quad \leq C N^{-(K+3) / 2} e^{-C^{\prime}|z-y|+C^{\prime}|y-w|}\left(1+|z|^{m}+|y|^{m}+|w|^{m}\right) .
\end{aligned}
$$

Hence, for each function $u \in \mathcal{S}$, one has

$$
\left\|A_{j, l, N}(u)\right\|_{L^{2}}=O\left(N^{-(K+3) / 2}\right) .
$$

In particular it is true of the functions $u_{k}$.

$A_{j, N}^{\prime}$ is the operator with kernel:

$$
\begin{aligned}
A_{j, N}^{\prime}(z, w)=\int_{\Omega_{N}} \Pi_{1}(z, y) & b_{j}(z, y) h\left(N^{-1 / 2} y\right) R_{K}(y, w, N) \mathrm{d} y \\
& \quad+\int \Pi_{1}(y, w) b_{j}(y, w) R_{K}(z, y, N) h\left(N^{-1 / 2} y\right) \mathrm{d} y .
\end{aligned}
$$

One has $h\left(N^{-1 / 2} y\right) \leq C N^{-1}|y|^{2}$, so there are constants $C>0, C^{\prime}>$ $0, m>0$ such that, on $\Omega_{N}^{3}$ :

$$
\begin{aligned}
& \left|\Pi_{1}(z, y) b_{j}(z, y) h\left(N^{-1 / 2} y\right) R_{K}(y, w, N)\right| \\
& \leq C N^{-(K+3) / 2} e^{-C^{\prime}|z-y|-C^{\prime}|y-w|}\left(1+|z|^{m}+|y|^{m}+|w|^{m}\right) .
\end{aligned}
$$


As usual we get, for every $k$, that

$$
\left\|A_{j, N}^{\prime}\left(u_{k}\right)\right\|_{L^{2}}=O\left(N^{-(K+3) / 2}\right) .
$$

$A_{N}^{\prime \prime}$ is the operator with kernel

$$
A_{N}^{\prime \prime}(x, z)=\int_{\Omega_{N}^{3}} R_{K}(x, y, N) h\left(N^{-1 / 2} y\right) R_{K}(y, z, N) \mathrm{d} y .
$$

Again there exist constants $C>0, C^{\prime}>0, m>0$ such that, on $\Omega_{N}^{3}$ :

$$
\begin{aligned}
& \left|R_{K}(z, y, N) h\left(N^{-1 / 2} y\right) R_{K}(y, w, N)\right| \\
& \quad \leq C N^{-K-3} e^{-C^{\prime}|z-y|-C^{\prime}|y-w|}\left(1+|z|^{m}+|y|^{m}+|w|^{m}\right) .
\end{aligned}
$$

To conclude, the $L^{2}$-norm of all the error terms is $O\left(N^{-(K+3) / 2}\right)$.

From this proposition we conclude that, at every well $P$, there exists an eigenvalue of $T_{N}(h)$ which has an asymptotic expansion in inverse powers of $N$, the dominant term being $N^{-1} \mu(P)$. In particular, the first eigenvalue of $T_{N}(h)$ is $O\left(N^{-1}\right)$.

\subsection{Positivity}

The following proposition implies that the first eigenfunctions only concentrate on the wells that are minimal:

Proposition 4.4. Let $\left(v_{N}\right)_{N \in \mathbb{N}}$ a sequence of normalized functions in $L^{2}(X)$. Suppose $v$ concentrates at a point $P_{0}$, on which $h$ vanishes. Then for each $\epsilon>0$ there exists $N_{0}$ and $C$ such that, if $N>N_{0}$,

$$
\left\langle v_{N}, h v_{N}\right\rangle \geq N^{-1} \mu\left(P_{0}\right)-C N^{-3 / 2+\epsilon} .
$$

Proof. Let $\delta<\frac{1}{2}$ be close to $\frac{1}{2}$. Let $\rho$ denote a normal map around $P_{0}$. Then the sequence $\left(w_{N}\right)_{N>0}=\left(\rho^{*} v_{N}\right)_{N>0}$ is such that $\left\|w_{N}\right\|_{L^{2}\left({ }^{c} B\left(0, N^{-\delta}\right)\right)}=$ $O\left(N^{-\infty}\right)$. Then one has as well:

$$
\begin{aligned}
& \left\|\Pi_{N} w_{N}\right\|_{L^{2}\left({ }^{c} B\left(0,2 N^{-\delta}\right)\right)}=O\left(N^{-\infty}\right) \\
& \left\|S_{N}^{P_{0}} w_{N}\right\|_{L^{2}\left({ }^{c} B\left(0,2 N^{-\delta}\right)\right)}=O\left(N^{-\infty}\right) .
\end{aligned}
$$

Using the Proposition 2.7, for $\delta$ close enough to $\frac{1}{2}$, if $\rho^{*} \Pi_{N}$ is a pull-back of $\Pi_{N}$ by $\rho$, one has $\left\|\left(S_{N}-\rho^{*} \Pi_{N}\right) v_{N}\right\| \leq C N^{-\frac{1}{2}+\epsilon}$. Hence,

$$
\left\|\left(S_{N}^{P_{0}}-\Pi_{N}\right) w_{N}\right\| \leq C N^{-\frac{1}{2}+\epsilon} .
$$


If $q$ is the Hessian of $h$ at $P_{0}$ read in the chosen coordinates, the spectrum of the model quadratic operator $\Pi_{N} q \Pi_{N}$ is known: one has

$$
\left\langle w_{N}, \Pi_{N} q \Pi_{N} w_{N}\right\rangle \geq N^{-1} \mu\left(P_{0}\right)\left\|\Pi_{N} w_{N}\right\|^{2} .
$$

Moreover, on $B\left(0,2 N^{-\delta}\right)$ the following holds: $C N^{-2 \delta} \geq h \geq q-C N^{-3 \delta}$.

Now, if $\delta$ is close enough to $\frac{1}{2}$, one has:

$$
\begin{aligned}
& \left\langle w_{N}, S_{N}^{P_{0}} h S_{N}^{P_{0}} w_{N}\right\rangle \\
& \geq\left\langle w_{N}, S_{N}^{P_{0}} q S_{N}^{P_{0}} w_{N}\right\rangle-C N^{-3 \delta} \\
& =\left\langle w_{N}, S_{N}^{P_{0}} q \Pi_{N} w_{N}\right\rangle+\left\langle w_{N}, S_{N}^{P_{0}} q\left(S_{N}^{P_{0}}-\Pi_{N}\right) w_{N}\right\rangle-C N^{-3 \delta} \\
& \geq\left\langle w_{N}, S_{N}^{P_{0}} q \Pi_{N} w_{N}\right\rangle-C N^{-2 \delta-\min \left(\delta, \frac{1}{2}-\epsilon\right)} \\
& =\left\langle w_{N}, \Pi_{N} q \Pi_{N} w_{N}\right\rangle+\left\langle w_{N},\left(S_{N}^{P_{0}}-\Pi_{N}\right) q \Pi_{N} w_{N}\right\rangle-C N^{-2 \delta-\min \left(\delta, \frac{1}{2}-\epsilon\right)} \\
& \geq\left\langle w_{N}, \Pi_{N} q \Pi_{N} w_{N}\right\rangle-C N^{-2 \delta-\min \left(\delta, \frac{1}{2}-\epsilon\right)} \\
& \geq N^{-1} \mu\left(P_{0}\right)-C N^{-2 \delta-\min \left(\delta, \frac{1}{2}-\epsilon\right)} .
\end{aligned}
$$

Choosing $\delta$ such that $\delta \geq \frac{1}{2}-\epsilon$ concludes the proof.

Remark 4.5. In the proof, it appears that the condition of concentration on $P_{0}$ can be slightly relaxed. We only used the fact that, for some fixed $\delta$ determined by the geometry of $M$ and by $\epsilon$, one has

$$
\left\|v_{N} 1_{\pi(x) \notin B\left(P_{0}, N^{-\delta}\right)}\right\|_{L^{2}}=O\left(N^{-\infty}\right) .
$$

Thus, this proposition could be used in a more general context.

\subsection{Uniqueness and spectral gap}

Proposition 4.6. Suppose $h$ satisfies the wells condition, and that there is only one well with minimal $\mu$. Then the approximate eigenvalues of proposition 4.2 associated to this well correspond to the first eigenvalue $\lambda_{N}$ of $T_{N}(h)$, namely, for every $K \in \mathbb{N}$, there holds:

$$
\left|\lambda^{K}(N)-\lambda_{N}\right|=O\left(N^{-(K+3) / 2}\right) .
$$

This eigenvalue is simple; moreover there exists $C>0$ such that, for $N$ large enough:

$$
\operatorname{dist}\left(\lambda_{N}, \operatorname{Sp}\left(T_{N}(h)\right) \backslash\left\{\lambda_{N}\right\}\right) \geq C N^{-1} .
$$


Proof. The proposition is equivalent to the claim that there exists $K$ such that the following is true: let $u_{K}(N)$ denote the approximate eigenvector of order $K$ associated to the well with minimal $\mu$. Let $F_{N}$ be the orthogonal complement of $u_{K}(N)$ in $H_{N}(X)$, and $p_{N}$ be the orthogonal projection from $H_{N}(X)$ to $F_{N}$. Then the operator $T_{N}^{\sharp}(h): F_{N} \rightarrow F_{N}$, defined as $T_{N}^{\sharp}(h)=p_{N} T_{N}(h)$, is bounded from below by $\lambda_{N}+C N^{-1}$.

Let $v_{N}$ be a sequence of normalized eigenvectors of $T_{N}^{\sharp}(h)$, and $\mu_{N}$ the sequence of associated eigenvalues. One has $T_{N}(h) v_{N}=\mu_{N} v_{N}+C_{N} u_{K}(N)$. Because $u_{K}(N)$ is a sequence of normalized functions and $S_{N}$ is bounded, the sequence $C_{N}$ is bounded.

Assume $\mu_{N}=O\left(N^{-1}\right)$. In this slightly different setting, we can adapt the proof of Proposition 3.1 using the fact that $u_{K}(N)$ is itself an almost eigenfunction of $T_{N}(h)$. There holds:

$$
T_{N}\left(h^{\star k}\right) v_{N}=\mu_{N}^{k} v_{N}+C_{N} \sum_{j=1}^{k} \mu_{N}^{j-1} \lambda_{N}^{k-j} u_{K}(N)+O\left(N^{-(K+3) / 2}\right) .
$$

We can proceed as in 3.1 but the induction process stops at $k=\frac{K+3}{2}$. One concludes that, for every $\epsilon>0$, the $L^{2}$ norm of $v_{N}$ is $O\left(N^{-\frac{K+3-\epsilon}{4}}\right)$ outside the union of balls centred at the vanishing points of $h$, and of radius $N^{-\frac{1}{2}+\frac{\epsilon}{K+3}}$.

In particular, if $P_{0}, P_{1}, \ldots, P_{d}$ denote the vanishing points of $h$, and $P_{0}$ is the only one with minimal $\mu$, one can decompose $v_{N}=v_{0, N}+v_{1, N}+$ $\ldots+v_{d, N}+O\left(N^{-(K+3-\epsilon) / 4}\right)$, where each sequence $v_{i, N}$ concentrates on $P_{i}$. The proposition 4.4 gives estimates for $v_{i, N}$ if $i \neq 0$. Indeed $\mu\left(P_{i}\right)>\mu\left(P_{0}\right)$ by construction, and $\lambda_{N} \leq N^{-1} \mu\left(P_{0}\right)+O\left(N^{-3 / 2}\right)$, so one can find $C>0$ small enough such that $N \lambda_{N}+C<\mu\left(P_{i}\right)$ for all $i \neq 0$ and for $N$ large enough. Then

$$
\left\langle v_{i, N}, S_{N} h S_{N} v_{i, N}\right\rangle \geq\left(\lambda_{N}+C N^{-1}\right)\left\|v_{i, N}\right\|_{2}^{2}
$$

Recall that $u_{K}(N)$ has an asymptotic expansion whose first term $u_{0}$ is the pull-backed ground state of the operator on the Bargmann space with quadratic symbol. This operator has a (fixed) nonzero specral gap. Moreover $\left\langle v_{0, N}, u_{K}(N)\right\rangle=O\left(N^{-(K+3-\epsilon) / 4}\right)$ because $v_{N}$ is orthogonal to $u_{K}(N)$ and $u_{K}(N)$ concentrates only at $P_{0}$. Then for $C$ strictly smaller than the spectral gap of the quadratic operator $T_{1}^{\text {flat }}\left(q_{0}\right)$ at $P_{0}$, one has for $N$ large

$$
\left\langle v_{0, N}, S_{N} h S_{N} v_{0, N}\right\rangle \geq\left(\lambda_{N}+C N^{-1}\right)\left\|v_{0, N}\right\|_{2}^{2} .
$$


The functions $v_{i, N}$ have disjoint supports, so that $\left\langle v_{i, N}, S_{N} h S_{N} v_{j, N}\right\rangle=$ $O\left(N^{-\infty}\right)$ whenever $i \neq j$, and $\left\|v_{N}\right\|_{2}^{2}=\sum_{j}\left\|v_{j, N}\right\|_{2}^{2}+O\left(N^{-(K+3-\epsilon) / 4}\right)$. Thus the two inequalities allow us to conclude when $K \geq 2$.

\subsection{End of the proof}

It remains to show that, in the case where only one well $P_{0}$ has minimal $\mu$, then the ground state is $O\left(N^{-\infty}\right)$ in a fixed neighbourhood of the other wells. Let $K \in \mathbb{N}$. We have constructed in Subsection 4.1 a sequence $\left(u_{K}(N)\right)_{N \in \mathbb{N}}$ which vanishes outside a fixed neighbourhood of $P_{0}$, and which is a sequence of approximate unit eigenvectors of $T_{N}(h)$, with approximate eigenvalue $\lambda_{K}(N)$. One has

$$
\lambda_{K}(N)=N^{-1} \mu\left(P_{0}\right)+O\left(N^{-3 / 2}\right),
$$

and

$$
\operatorname{dist}\left(\lambda_{K}(N), \operatorname{Sp}\left(T_{N}(h)\right)=O\left(N^{-(K+3) / 2}\right) .\right.
$$

Moreover we proved in Subsection 4.3 that there can be only one eigenvalue of $T_{N}(h)$ in $\left[0, N^{-1}\left(\mu\left(P_{0}\right)+C\right)\right]$ for some $C$, and that this eigenvalue is simple. Hence, denoting $\lambda_{\infty}(N)$ this sequence of eigenvalues, one has

$$
\lambda_{\infty}(N)=\min \operatorname{Sp}\left(T_{N}(h)\right),
$$

and

$$
\left|\lambda_{\infty}(N)-\lambda_{K}(N)\right|=O\left(N^{-(K+3) / 2}\right) .
$$

Let $U_{\infty}(N)$ denote a sequence of unit eigenvectors associated to $\lambda_{\infty}(N)$, and decompose $u_{K}(N)=c(N) U_{\infty}(N)+w_{K}(N)$, where $w_{K}(N) \perp U_{\infty}(N)$. Then

$$
\left(T_{N}(h)-\lambda_{\infty}(N)\right) w_{K}(N)=O\left(N^{-(K+3) / 2}\right) .
$$

The operator $T_{N}(h)-\lambda_{\infty}(N)$ is invertible on $U_{\infty}(N)^{\perp}$ and its inverse has a norm bounded by $N$, so $w_{K}(N)=O\left(N^{-(K+1) / 2}\right)$. Since both $u_{K}(N)$ and $U_{\infty}(N)$ are normalized, one has $c(N) \rightarrow 1$.

Finally, if $V$ is a neighbourhood of another well, then $u_{K}(N)$ is zero on $V$, so that

$$
\left\|U_{\infty}(N)\right\|_{L^{2}(V)}=\left\|w_{K}(N)\right\|_{L^{2}(V)}=O\left(N^{-(K+1) / 2}\right) .
$$

This concludes the proof. 


\section{Eigenvalues in a scaled window}

This section is devoted to the proof of Theorem B. The method of proof is very similar to that of Theorem A: we will exhibit approximate eigenvectors, then show that they cover the low-energy spectrum.

\subsection{Approximate eigenvectors}

In the proof of the Proposition 4.2, the first guess for an approximate eigenvector of $T_{N}(h)$ was the first eigenvector of the model quadratic operator at one of the wells. If, instead of the first eigenvector, we start from any eigenvector of the model quadratic operator, we can proceed the same way; however the recursion stops after one step, in general.

Proposition 5.1. Let $P \in M$ on which $h$ cancels, and $Q$ be a model quadratic operator in some normal map $\rho$ around $P$. Let $\lambda$ be an eigenvalue of $Q$ and $E_{\lambda}$ the corresponding eigenspace. Then one can find a suitable orthonormal basis $\left(v_{1}, \ldots, v_{d}\right)$ of $E_{\lambda}$, functions $\left(w_{1}, \ldots, w_{d}\right)$ in $\mathcal{S}\left(\mathbb{C}^{n}\right)$ and real numbers $\left(b_{1}, \ldots, b_{d}\right)$ such that, for any integer $i \in[1, d]$, the function

$$
\tilde{v}_{i}(N): \rho(z, \theta) \mapsto N^{n} e^{i N \theta}\left(v_{i}\left(N^{1 / 2} z\right)+N^{-1 / 2} w_{i}\left(N^{1 / 2} z\right)\right)
$$

is such that

$$
S_{N} h S_{N} \tilde{v}_{i}(N)=N^{-1} \lambda+N^{-3 / 2} b_{i}+O\left(N^{-2}\right),
$$

Moreover, if $\operatorname{dim} E_{\lambda}=1$, then if $u_{0}$ is an eigenvector of $Q$, one can find a sequence of Schwartz functions $\left(u_{k}\right)_{k \geq 1}$, orthogonal to $u_{0}$, and a sequence of real numbers $\left(\lambda_{k}\right)_{k \geq 1}$, such that, for every $K>0$, the function

$$
u_{K}(N): \rho(z, \theta) \mapsto N^{n} e^{i N \theta} \sum_{k=0}^{K} N^{-k / 2} u_{k}\left(N^{1 / 2} z\right)
$$

is such that

$$
S_{N} h S_{N} u_{K}(N)=N^{-1} \lambda+N^{-1} \sum_{k=1}^{K / 2} N^{-k} \lambda_{k}=O\left(N^{-(K+3) / 2}\right) .
$$

Proof. Recall from Proposition 4.2 that one can find an approximate eigenvector at any order, starting from the ground state $u_{0}$ of $Q$.

Let now $u_{0}$ denote an arbitrary eigenfunction of $Q$, which still belongs to $\mathcal{D}$. Let $\lambda$ be the associated eigenvalue. When $\lambda$ is simple, the operator $Q-\lambda$ has a continuous inverse on $u_{0}^{\perp}$, so one can solve equation (77) at any 
order. Observe that $u_{0}$ is either even or odd, so that only negative integer powers of $N$ remain in the expansion of the eigenvalue.

If $Q-\lambda$ is not invertible on $u_{0}^{\perp}$, the equation (7) can still be solved for $K=1$ if $u_{0}$ is one of the vectors of a convenient basis of $E_{\lambda}$; but the construction fails at higher orders. Consider an orthonormal basis $\left(v_{1}, \ldots, v_{L}\right)$ of the eigenspace $E_{\lambda}$. Suppose $u_{0}=v_{l}$. The equation (7) reads:

$$
(Q-\lambda) u_{1}+J_{1} u_{0}=\lambda_{1} u_{0} .
$$

Taking the scalar product with $u_{0}$ yields $\lambda_{1}(l)=\left\langle v_{l}, J_{1} v_{l}\right\rangle$. But we also need to check that $0=\left\langle v_{l}, J_{1} v_{j}\right\rangle$ for $l \neq j$. This is done by choosing an orthogonal basis in which the corestriction of $J_{1}$ on $E_{\lambda}$ is diagonal (recall $J_{1}$ is symmetric and $E_{\lambda}$ is finite-dimensional). One can then find $u_{1}(l)$ in $E_{\lambda}^{\perp}$. The proof of the error estimate is the same. To conclude we let $b_{l}=\lambda_{1}(l)$ and $w_{l}=u_{1}(l)$.

Once the $K=1$ step is done, the basis $\left(v_{1}, \ldots, v_{L}\right)$ is fixed. Let us try to solve equation (7) with $u_{0}=v_{1}$, for $K=2$. We write

$$
(Q-\lambda) u_{2}+J_{2} u_{0}+J_{1} u_{1}=\lambda_{2} u_{0}+\lambda_{1} u_{1} .
$$

Taking the scalar product with $u_{0}$ yields $\lambda_{2}$ as previously:

$$
\lambda_{2}=\left\langle u_{0}, J_{2} u_{0}\right\rangle+\left\langle u_{0}, J_{1} u_{1}\right\rangle .
$$

Now recall $u_{1}$ is orthogonal to $E_{\lambda}$. If $v$ denotes an element of $E_{\lambda}$ orthogonal to $u_{0}$, then one must check

$$
\left\langle v, J_{2} u_{0}\right\rangle+\left\langle v, J_{1} u_{1}\right\rangle=0 .
$$

This equation does not hold in general, hence the obstruction.

\subsection{Uniqueness}

Let $C^{\prime}>0$, and $N \in \mathbb{N}$. Consider the set $e_{N}$ of approximate eigenvectors in Proposition [5.1, such that $\lambda<C^{\prime}$. Then $E_{N}=\operatorname{span}\left(e_{N}\right)$ is a subspace of $L^{2}(X)$, with small energy: there exists $C_{1}$ such that, for every $N$,

$$
\max \left\{\left\langle u, T_{N}(h) u\right\rangle, u \in E_{N},\|u\|_{2}^{2}=1\right\}<C^{\prime} N^{-1}+C_{1} N^{-\frac{3}{2}} .
$$

We claim that, reciprocally, any function approximately orthogonal with $E_{N}$ has an energy bounded from below: 
Proposition 5.2. Let $C^{\prime}>0$. There exists $\epsilon_{0}>0$ and a function $\epsilon \mapsto$ $N_{0}(\epsilon)$ such that, for $0<\epsilon<\epsilon_{0}$, the following is true. Let $v_{N}$ be a normalized eigenfunction of $T_{N}(h)$, with associated eigenvalue $\lambda_{N}$, and suppose that the angle between $v_{N}$ and $E_{N}$ is greater than $\cos ^{-1}(\epsilon)$, that is, for every $u \in E_{N}$ normalized, one has $\left|\left\langle u, v_{N}\right\rangle\right|<\epsilon$. Then for $N \geq N_{0}(\epsilon)$, one has

$$
\lambda_{N} \geq\left(C^{\prime}-\epsilon\right) N^{-1} \text {. }
$$

\section{Proof.}

Let $P_{0}, \ldots, P_{d}$ denote the points at which $h$ cancels. If $\lambda_{N}=O\left(N^{-1}\right)$, then $v_{N}$ concentrates on the $P_{i}^{\prime}$ 's. We decompose $v_{N}=v_{0, N}+v_{1, N}+\ldots+$ $v_{d, N}+O\left(N^{-\infty}\right)$, where each $v_{i, N}$ concentrates only on $P_{i}$.

Let $\rho_{i}$ be a normal map associated with $P_{i}$, and $q_{i}$ the Hessian of $h$ at $P_{i}$ read in the map $\rho_{i}$. Let $E_{i, N}$ be the span of eigenfunctions of $T_{N}^{\text {flat }}\left(q_{i}\right)$ whose eigenvalues are less than $C^{\prime} N^{-1}$. Then for $N$ large, for every normalized $u \in E_{i, N}$, one has $\left|\left\langle\rho_{i}^{*} v_{i, N}, u\right\rangle\right| \leq 2 \epsilon$. Indeed functions in $E_{N}$ are $N^{-1 / 2}$-close to sums of pull-backs of functions in $E_{i, N}$.

Hence, for $N$ large enough,

$$
\left\langle\rho_{i}^{*} v_{i, N}, \Pi_{N}\left(q_{i}-C^{\prime} N^{-1}\right) \Pi_{N} \rho_{i}^{*} v_{i, N}\right\rangle \geq-C^{\prime} N^{-1}\left(4 \epsilon^{2}\right) .
$$

Since $v_{i, N}$ concentrates on $P_{i}$, one can deduce that, for $N$ large enough,

$$
\left\langle v_{i, N}, S_{N} h S_{N} v_{i, N}\right\rangle \geq C^{\prime} N^{-1}\left\|v_{i, N}\right\|^{2}-C^{\prime} N^{-1}\left(5 \epsilon^{2}\right),
$$

hence

$$
\left\langle v_{N}, S_{N} h S_{N} v_{N}\right\rangle \geq C^{\prime} N^{-1}-C^{\prime} N^{-1}\left(5(d+2) \epsilon^{2}\right) .
$$

To conclude, we let $\epsilon_{0}=\frac{1}{5(d+2) C^{\prime}}$. Then for every $\epsilon<\epsilon_{0}$, for $N$ large enough,

$$
\left\langle v_{N}, S_{N} h S_{N} v_{N}\right\rangle \geq\left(C^{\prime}-\epsilon\right) N^{-1}
$$

To conclude the proof of Theorem $B$, if the rank of the spectral projector of $T_{N}(h)$ with interval $\left[0, C N^{-1}\right]$ was greater than $K$, then one could find an eigenfunction of $T_{N}(h)$ which forms an angle greater than $\cos ^{-1}\left(N^{-1}\right)$ with $E_{N}$, and with eigenvalue less than $C N^{-1}$. This is absurd since $C<C^{\prime}$.

\section{Acknowledgements}

The author thanks N. Anantharaman and L. Charles for their help and encouragement while writing this article. 


\section{Appendix : a proof for the off-diagonal estimate}

This last section is an appendix about Proposition 2.3. As we already explained, the knowledge of the result is sufficient for our needs. However, as this proposition appears in [13, it is stated in a case that is much more general than prequantum bundles on Kähler manifolds.

In this specific setting, and with a more direct approach, we propose to show a different version of this estimate, with a somewhat stronger estimate on the remainder (see Proposition A.8). We also replace the normal maps of Definition 2.2 with Heisenberg maps, satisfying different assumptions. This version could be of use in situations where it is crucial that the local map is a biholomorphism.

The proof relies on the theory of Fourier Integral Operators with complexvalued phase functions, in the sense of Hörmander ([22], section 7.8). Indeed, we will follow the lines of [33] (restricting ourselves to exact Kähler structures), which gives asymptotics at a shrinking scale; we modify the proof in order to estimate the remainder at a fixed scale, recovering results from [10, 4].

The starting point in [33] is the study by Boutet de Monvel and Sjöstrand [8] of the general Szegö projector (Definition 1.2). The structure of the Szego" projector, for the boundary of a relatively compact open set, has been subject to a thorough study $([23,24,25,6,6,7])$. Under the assumption of strong pseudo-convexity, which is verified for the unit ball $D$ of $L^{*}$, the boundary of $D$ inherits a Riemannian metric from the Levi form (which is identical to the one we use in this paper). The projector $S$ is then a Fourier Integral Operator with complex phase, in the sense of Hörmander [22]:

Proposition A.1 (8]). Let $Y$ be the boundary of a strongly pseudo-convex, relatively compact open set in a complex manifold. Then there exists a skewsymmetric almost holomorphic complex phase function $\psi \in C^{\infty}(Y \times Y)$ (in the sense of [22]), whose critical set is $\operatorname{diag}(Y)$, and a classical symbol

$$
s \sim \sum_{i} t^{n-i} s_{i} \in C^{\infty}\left(Y \times Y \times \mathbb{R}^{+}\right),
$$

such that the Schwartz kernel of the Szegö projector on $Y$ is

$$
S(x, y)=\int_{0}^{+\infty} e^{i t \psi(x, y)} s(x, y, t) \mathrm{d} t+E(x, y),
$$

where the function $E$ is smooth. Moreover the principal symbol $s_{0}$ is such that $s_{0}^{2}=h_{\psi}^{-1}$, where $h_{\psi}(x, y)$ is the Hessian of the function

$$
Y \times \mathbb{R}^{+} \ni(z, \sigma) \mapsto \psi(x, z)+\sigma \psi(z, y)
$$


at the critical point (which is unique and lies in a complex extension of $\left.Y \times \mathbb{R}^{+}\right)$.

In this setting, "almost holomorphic" means that, near the diagonal $z=w \in Y$, one has $\bar{\partial}_{z} \psi(z, w)=O\left(|z-w|^{\infty}\right)$. The fact that the function $(z, \sigma) \mapsto \psi(x, z)+\sigma \psi(z, y)$ has exactly one critical point in the complex extension of $Y \times \mathbb{R}^{+}$, with nondegenerate Hessian, is encoded in the requirements on $\psi$ to be a complex phase function in the sense of Hörmander.

In the specific case where $X$ is a circle bundle over $M$, one can use the microlocal information on $S$ to deduce the asymptotics of its Fourier components $S_{N}$. Indeed, the $N$-th Fourier component of a smooth function on a compact set has a sup norm bounded by $O\left(N^{-\infty}\right)$. Thus, one has

$$
S_{N}(x, y)=\iint \exp \left(i t \psi\left(x, r_{\eta} y\right)+i N \eta\right) s\left(x, r_{\eta} y, t\right) \mathrm{d} t \mathrm{~d} \eta+E_{N}(x, y),
$$

where $\left\|E_{N}\right\|_{L^{\infty}}=O\left(N^{-\infty}\right)$. Here, as in the introduction, $r_{\eta}$ denotes the circle action on $X$.

As announced, we will deal with a less restrictive class of local maps than the normal maps of Definition 2.2. Because we are dealing with exact Kähler manifolds, as opposed to the more general almost complex structure, we slightly modify the definition of [33]:

Definition A.2. Let $P_{0} \in M$. Let $U$ be a neighbourhood of 0 in $\mathbb{C}^{n}$ and $V$ be a neighbourhood of $P_{0}$ in $M$.

A smooth diffeomorphism $\rho: U \times \mathbb{R} \rightarrow \pi^{-1}(V)$ is said to be an Heisenberg map or map of Heisenberg coordinates under the following conditions:

- $\pi(\rho(0,0))=P_{0}$;

- $\rho^{*} \omega\left(P_{0}\right)=\omega_{0}(0)$.

- $\bar{\partial} \rho=0$.

- $\rho(m, \theta)=r_{\theta} \rho(m, 0)$.

The crucial point is that, in these coordinates, the phase $\psi$ from the Boutet-Sjöstrand theorem reads, for all $(z, \theta)$ and $(w, \phi)$ in the domain of $\rho$ (cf. [33], equation 61):

$$
\psi(\rho(z, \theta), \rho(w, \phi))=i\left[1-A(z, w) e^{i(\theta-\phi)}\right],
$$

Here, the 2-jet of $A$ is known at the origin ([33], Lemma 2.4):

$$
A(z, w)=1-\frac{1}{2}|z-w|^{2}+i \Im(z \cdot \bar{w})+O\left(|z|^{3},|w|^{3}\right) .
$$


We will need to control the off-diagonal behaviour of $A$. Recall

$$
\Pi_{1}:(z, w) \mapsto \frac{1}{\pi^{n}} \exp \left(-\frac{1}{2}|z-w|^{2}+i \Im(z \cdot \bar{w})\right) .
$$

Up to a reduction of the definition set of $\rho$, the usual logarithm is welldefined, and we can define $R_{A}$ as the unique function such that $A / \Pi_{1}=$ $\pi^{n} e^{R_{A}}$.

Proposition A.3. The two following estimates hold as $z, w \rightarrow 0$ :

$$
\begin{aligned}
& \Re\left(R_{A}\right)(z, w)=O\left(|z-w|^{2}(|z|+|w|)\right) \\
& \Im\left(R_{A}\right)(z, w)=O\left(|z-w|\left(|z|^{2}+|w|^{2}\right)\right) .
\end{aligned}
$$

In particular, up to a restriction of the Heisenberg map $\rho$ to a smaller neighbourhood of $P_{0}$, one has, for every $z$ and $w$ in the domain of $\rho$ :

$$
\left|A / \Pi_{1}\right|(z, w) \leq \pi^{n} e^{\frac{1}{4}|z-w|^{2}} .
$$

Proof. The functions $A$ and $\pi^{n} \Pi_{1}$ are equal up to order 2 at $P_{0}$, so that $R_{A}(z, w)=O\left(|z|^{3},|w|^{3}\right)$.

The two functions $A$ and $\pi^{n} \Pi_{1}$ are both smooth and are equal to 1 on the diagonal. Moreover the first derivatives of both $\Re(A)$ and $\Re\left(\Pi_{1}\right)$ vanish on the diagonal. For $\Pi_{1}$ this is a straightforward computation. For $A$ it comes from the fact that $\psi$ is a complex phase function whose critical set is the diagonal. It is also a natural consequence of the fact that $\partial_{1} A(z, z)=-\frac{1}{2} \partial \phi(z)$ and $\bar{\partial}_{1} A(z, z)=\frac{1}{2} \partial \phi(z)$, where $\phi$ is a complex potential: $i \partial \bar{\partial} \phi=\omega$. Hence there is a constant $C$ such that, for every $z$ and $w$ in the domain of $\rho$, there holds:

$$
\begin{aligned}
& \left|\Im\left(A-\pi^{n} \Pi_{1}\right)(z, w)\right| \leq C|z-w|\left(|z|^{2}+|w|^{2}\right) \\
& \left|\Re\left(A-\pi^{n} \Pi_{1}\right)(z, w)\right| \leq C|z-w|^{2}(|z|+|w|) .
\end{aligned}
$$

From which we deduce that

$$
\begin{aligned}
& \left|\Re\left(\left(A-\pi^{n} \Pi_{1}\right)^{2}\right)(z, w)\right| \leq C|z-w|^{2}(|z|+|w|) \\
& \left|\Im\left(\left(A-\pi^{n} \Pi_{1}\right)^{2}\right)(z, w)\right| \leq C|z-w|^{3} \\
& \left|A-\pi^{n} \Pi_{1}\right|^{3} \leq|z-w|^{3} .
\end{aligned}
$$

Now

$$
R_{A}=\log \left(A / \pi^{n} \Pi_{1}\right)=\frac{A-\pi^{n} \Pi_{1}}{\pi^{n} \Pi_{1}}-\frac{1}{2}\left(\frac{A-\pi^{n} \Pi_{1}}{\pi^{n} \Pi_{1}}\right)^{2}+O\left(\left(\frac{A-\pi^{n} \Pi_{1}}{\pi^{n} \Pi_{1}}\right)^{3}\right) .
$$


Taking the real and imaginary part of this equation, one deduces

$$
\begin{aligned}
& \Re\left(R_{A}\right)(z, w)=O\left(|z-w|^{2}(|z|+|w|)\right) \\
& \Im\left(R_{A}\right)(z, w)=O\left(|z-w|\left(|z|^{2}+|w|^{2}\right)\right) .
\end{aligned}
$$

In particular,

$$
\left|A / \Pi_{1}\right|(z, w) \leq \pi^{n} e^{C|z-w|^{2}(|z|+|w|)} .
$$

Reducing the domain of the Heisenberg map $\rho$ to a smaller neighbourhood of $P_{0}$, one gets, for every $z$ and $w$ in the domain of $\rho$ :

$$
\left|A / \Pi_{1}\right|(z, w) \leq \pi^{n} e^{\frac{1}{4}|z-w|^{2}} .
$$

In fact, the symbol $s$ of the operator can also be chosen to be very simple in the given coordinates:

Proposition A.4. In Heisenberg coordinates, the symbol s of $S$ in proposition A.1 can be chosen to be factorized as:

$$
s(\rho(z, \theta), \rho(w, \phi), t)=e^{-i n(\theta-\phi)} \xi(z, w, t),
$$

where

$$
\xi(z, w, t) \sim \sum_{k=0}^{+\infty} t^{n-k} \xi_{k}(z, w)
$$

and where each $\xi_{k}$ is a smooth function. Moreover the principal symbol $\xi_{0}$ does not vanish in a neighbourhood of $\operatorname{diag}(M)$.

Proof. The expression of the phase $\psi$ in local coordinates gives immediatly that any derivative of order $\geq 2$ of the function $(t, z, \theta, w, \phi) \mapsto$ $t \psi(\rho(z, \theta), \rho(w, \phi))$ is of the form $e^{i(\theta-\phi)} f(z, w, t)$ where $f$ is constant or linear wrt $t$. It follows that $h_{\psi}(\rho(z, \theta), \rho(w, \phi))=e^{2 i n(\theta-\phi)} g(z, w)$ for some function $g$. Hence, we can write $s_{0}(\rho(z, \theta), \rho(w, \phi))=e^{-i n(\theta-\phi)} \xi_{0}(z, w)$ for some smooth function $\xi_{0}$. Of course, any partial derivative of $s_{0}$ is also, in local coordinates, of the form $e^{-i n(\theta-\phi)} f(z, w)$ for some function $f$.

Let us assume that for $k \leq K$, each function $s_{k}$ reads in local coordinates as $e^{i n(\theta-\phi)} \xi_{k}(z, w)$ for some smooth function $\xi_{k}$. The coefficient $s_{K+1}$ can be derived from $\left(s_{i}\right)_{i \leq K}$ via a stationary phase lemma, in which the differential operators come from the Taylor expansion of $\psi$. Thus, $s_{K+1}$ is a priori of the form

$$
s_{K+1}(\rho(z, \theta), \rho(w, \phi))=e^{-i n(\theta-\phi)}\left(\sum_{j=-C}^{C} e^{i k(\theta-\phi)} \xi_{K+1, j}(z, w)\right),
$$


where $C$ is finite (but depends on $K$ ) and the $\xi_{K+1, j}$ are smooth functions.

We can get rid of all coefficients except $j=0$ by adding a convenient multiple of $\psi$. Indeed, the operator with symbol $(f+\psi g) t^{k}$ is equal, after integration by parts, to the operator with symbol $f t^{k}+i k g t^{k-1}$, modulo a smoothing operator. For instance, replacing $s_{K+1}$ with $s_{K+1}+$ $e^{-i(\theta-\phi)} \xi_{K+1,1} a(z, w) \psi$ eliminates the $j=1$ term.

We conclude by induction.

The $N$-th Fourier component $S_{N}$ of the Szegö projector at a point $(x, y)$ reads

$$
S_{N}(x, y)=\iint \exp \left(i t \psi\left(x, r_{\eta} y\right)+i N \eta\right) s\left(x, r_{\eta} y, t\right) \mathrm{d} t \mathrm{~d} \eta+O\left(N^{-\infty}\right) .
$$

A change of variables leads to

$$
S_{N}(x, y)=N \iint \exp \left(i N\left(t \psi\left(x, r_{\eta} y\right)+\eta\right)\right) s\left(x, r_{\eta} y, N t\right) \mathrm{d} t \mathrm{~d} \eta+O\left(N^{-\infty}\right) .
$$

If $x$ and $y$ belong to different fibres, the phase $t \psi\left(x, r_{\eta} y\right)+\eta$ has no critical point, so $S_{N}(x, y)=O\left(N^{-\infty}\right)$; this estimation is uniform outside a neighbourhood of $\pi^{-1}(\operatorname{diag}(M))$.

Using the local expression of the phase, one can derive as in [33] an expression for $S_{N}$ at a neighbourhood of size $N^{-1 / 2}$ of the diagonal. Let $\Omega_{N} \subset \mathbb{C}^{n} \times \mathbb{R}$ be the set of those $(z, \theta)$ such that $(z / \sqrt{N}, \theta / N)$ belongs to the domain of $\rho$.

Proposition A.5 ([33], Theorem 3.1). There exists a sequence $\left(b_{k}\right)_{k \in \mathbb{N}}$ of polynomials on $\mathbb{R}^{4 n}$, such that each $b_{k}$ is of same parity as $k$, and a smooth function $R_{K}$ on $\mathbb{C}^{2 n} \times \mathbb{N}$, bounded on the compact sets of $\mathbb{C}^{2 n}$ independently of the second variable, such that for all $N$, for all $(z, w, \theta, p h i) \in \Omega_{N}^{2} \times \mathbb{R}^{2}$, there holds

$$
\begin{array}{r}
N^{-n} e^{i(\phi-\theta)} S_{N}\left(\rho\left(\frac{z}{\sqrt{N}}, \frac{\theta}{N}\right),\left(\frac{w}{\sqrt{N}}, \frac{\phi}{N}\right)\right) \\
=\Pi_{1}(z, w)\left(1+\sum_{k=1}^{K} N^{-k / 2} b_{k}\left(z, w, P_{0}\right)+N^{-(K+1) / 2} R_{K}(z, w, N)\right) \\
+O\left(N^{-\infty}\right) .
\end{array}
$$

Here, $\Pi_{1}$ is the kernel of the projector on the Bargmann space, as in equation (1).

Remark A.6. The next step is Proposition A.8, an estimate for $R_{K}$ that is valid in all of $\Omega_{N}^{2}$. For this, we have to keep the $O\left(N^{-\infty}\right)$ term outside. 
In [33], the $O\left(N^{-\infty}\right)$ term is absorbed into $R_{K}$, without altering the property that $R_{K}$ is bounded on compact sets independently on $N$. However, if an estimate such that the one in Proposition A.8 did hold without the supplementary $O\left(N^{-\infty}\right)$ term, then one could deduce exponential estimates for the off-diagonal of $S_{N}$, that is, $\left|S_{N}(x, y)\right| \leq e^{-c N|x-y|^{2}}$ for some $C$. Such results are indeed known [4] but cannot be obtained via the BoutetSjöstrand parametrix because the Boutet-Guillemin construction [7] adapts the Szegő kernel parametrix to the more general case of almost Kähler manifolds, where exponential estimates for the off-diagonal of $S_{N}$ fail to hold [12].

The method of proof for the last proposition can be in fact adapted to compute $S_{N}$ in a fixed neighbourhood of a point on the diagonal, giving a result close to the Theorem 4.18 of [28, which also appears in [9, 4]. Recall

$$
S_{N}(x, y)=N \iint \exp \left(i N\left(t \psi\left(x, r_{\eta} y\right)+\eta\right)\right) s\left(x, r_{\eta} y, N t\right) \mathrm{d} t \mathrm{~d} \eta+O\left(N^{-\infty}\right) .
$$

Replacing $\psi$ and $s$ by their expressions we get, after a change of variables,

$$
\begin{aligned}
& S_{N}(\rho(z, \theta), \rho(w, \phi)) \\
& =N e^{i N(\theta-\phi)} \iint e^{-N\left(t\left(1-A(z, w) e^{i \eta}\right)-i \eta\right)} e^{i n \eta} \xi(z, w, N t) \mathrm{d} t \mathrm{~d} \eta+O\left(N^{-\infty}\right) .
\end{aligned}
$$

We cannot use the stationary phase lemma, except if $z=w$, because the phase has no critical points. But $\psi$ and $s$ depend holomorphically on $e^{i \eta}$. Thus, we can replace this integral, which is a contour integral on the unit circle, with an integral on the circle of radius $|A(z, w)|$ in order to get a phase with a critical point. This corresponds to formally changing $\eta$ into $\eta-i \log (|A(z, w)|)$ in the computations. The integral now reads

$$
\begin{aligned}
& S_{N}(\rho(z, \theta), \rho(w, \phi))= \\
& N A(z, w)^{N} e^{i N(\theta-\phi)} \iint e^{-N\left(t\left(1-e^{i \eta}\right)-i \eta\right)} e^{i n \eta} \frac{\xi(z, w, N t)}{A(z, w)^{n}} \mathrm{~d} t \mathrm{~d} \eta+O\left(N^{-\infty}\right) .
\end{aligned}
$$

The last part of the product can now be computed using a stationary phase lemma, and the fact that $\xi$ is a classical symbol. Hence, we recover a result similar to [28, 10, 4]:

Proposition A.7. There exists a neighbourhood $V$ of $(\pi, \pi)^{-1} \operatorname{diag}(M)$ in $X \times X$ such that one has, in local Heisenberg coordinates around a point 
$P_{0} \in \operatorname{diag}(X)$ with values in $V$, and for each integer $K$ :

$$
\begin{aligned}
& S_{N}(\rho(z, \theta), \rho(w, \phi)) \\
=N^{n} e^{i N(\theta-\phi)} A(z, w)^{N}\left(\sum_{j=0}^{K} N^{-j} B_{j}\left(z, w, P_{0}\right)+N^{-(K+1)} r_{K}\left(z, w, N, P_{0}\right)\right) & +O\left(N^{-\infty}\right) .
\end{aligned}
$$

Each $B_{j}$ is smooth and $B_{0}$ is $\frac{1}{\pi^{n}}$ on the diagonal. Moreover, $r_{K}$ is bounded in a compact subset of the domain of definition of $\rho$, independently of $P_{0}$ and $N$.

On the diagonal set, $B_{0}\left(z, z, P_{0}\right)=\frac{1}{\pi^{n}}$ because $S_{N}$ is a projector.

Since, in a neighbourhood small enough of the diagonal, one has

$$
|A(z, w)| \leq 1-\frac{1}{4}|z-w|^{2},
$$

equation (9) can be deduced from equation (10). This way, we obtain an estimate on the remainder:

Proposition A.8. In the equation (91), there exist $C$ and $m$ such that the remainder $R_{K}$ satisfies, for every $N$, for every $z$ and $w$ in $\Omega_{N}$, the inequality:

$$
\left|R_{K}\left(z, w, N, P_{0}\right)\right| \leq C e^{\frac{1}{4}|z-w|^{2}}\left(1+|z|^{m}+|w|^{m}\right) .
$$

Proof. Rescaling the formula (10) yields:

$$
\begin{aligned}
& N^{-n} e^{i(\phi-\theta)} S_{N}\left(\rho\left(\frac{z}{\sqrt{N}}, \frac{\theta}{N}\right),\left(\frac{w}{\sqrt{N}}, \frac{\phi}{N}\right)\right) \\
&= A\left(\frac{z}{\sqrt{N}}, \frac{w}{\sqrt{N}}\right)^{N}\left(\sum_{j=0}^{K} N^{-j} B_{j}\left(\frac{z}{\sqrt{N}}, \frac{w}{\sqrt{N}}\right)+N^{-(K+1)} r_{K}\left(\frac{z}{\sqrt{N}}, \frac{w}{\sqrt{N}}, N\right)\right) \\
&+O\left(N^{-\infty}\right)
\end{aligned}
$$

The functions $B_{j}$ are smooth, and $r_{K}$ is bounded independently of $N$. Thus, applying a Taylor expansion at the origin, there exist polynomials 
$b_{j}^{s}$, and a function $r_{K}^{s}$ with polynomial growth independent of $N$, such that

$$
\begin{aligned}
& N^{-n} e^{i(\phi-\theta)} S_{N}\left(\rho\left(\frac{z}{\sqrt{N}}, \frac{\theta}{N}\right),\left(\frac{w}{\sqrt{N}}, \frac{\phi}{N}\right)\right) \\
&=A\left(\frac{z}{\sqrt{N}}, \frac{w}{\sqrt{N}}\right)^{N}\left(\sum_{j=0}^{2 K+1} N^{-j / 2} b_{j}^{s}(z, w)+N^{-(K+1)} r_{K}^{s}(z, w, N)\right) \\
&+O\left(N^{-\infty}\right) .
\end{aligned}
$$

Let again $R_{A}$ be such that $A(z, w)=\pi^{n} \Pi_{1}(z, w) e^{R_{A}(z, w)}$. We wish to control, for any integer $N$, the Taylor expansion at zero of

$$
g_{N}:(z, w) \mapsto e^{N R_{A}\left(\frac{z}{\sqrt{N}}, \frac{w}{\sqrt{N}}\right)} .
$$

For every multi-index $\alpha$, the derivative of degree $\alpha$ of $g_{N}$ is a sum of terms of the form

$$
e^{N R_{A}\left(\frac{z}{\sqrt{N}}, \frac{w}{\sqrt{N}}\right)} \prod_{i=1}^{4 n} N^{1-\frac{1}{2}\left|\beta_{i}\right|} \partial_{i}^{\beta_{i}} R_{A}\left(\frac{z}{\sqrt{N}}, \frac{w}{\sqrt{N}}\right),
$$

where each index $\beta_{i}$ is nonzero and $\sum \beta_{i}=\alpha$.

Recall that $A$ and $\pi^{n} \Pi_{1}$ coincide up to order 2 at the origin. In particular, the derivatives of order less than 2 of $R_{A}$ vanish at the origin. It follows that a term of the form above is nonzero at the origin only if, for each $1 \leq i \leq 4 n$, there holds $\beta_{i} \geq 3$. In particular, for each $\alpha$ there holds

$$
\partial^{\alpha} g_{N}(0,0)=O\left(N^{-|\alpha| / 6}\right) .
$$

Moreover, $\partial^{\alpha} g_{N}(0,0)$ is always a polynomial in $N^{-1 / 2}$.

As we want to write an expansion with a remainder in $O\left(N^{-K-1}\right)$, let us consider the Taylor expansion of $g_{N}$ at order $6 K+5$. To control the remainder, we make use again of the fact that $R_{A}$ is smooth on a compact set and that $R_{A}(z, w)=O\left(|z|^{3},|w|^{3}\right)$ at the origin. If $\beta_{i}=1$, then there is a constant $C$ such that, for every $(z, w)$ and every $N$, one has

$$
\left|\partial_{i}^{\beta_{i}} R_{A}\left(\frac{z}{\sqrt{N}}, \frac{w}{\sqrt{N}}\right)\right| \leq C N^{-1}\left(|z|^{2}+|w|^{2}\right) .
$$

Similarly, if $\beta_{i}=2$, there exists a constant $C$ such that, for every $(z, w)$ and every $N$, one has

$$
\left|\partial_{i}^{\beta_{i}} R_{A}\left(\frac{z}{\sqrt{N}}, \frac{w}{\sqrt{N}}\right)\right| \leq C N^{-1 / 2}(|z|+|w|) .
$$


If $\beta_{i} \geq 3$ we simply use the fact that the function $\partial_{i}^{\beta_{i}} R_{A}$ is bounded on its set of definition. It follows that for every $\alpha$ there exist $m$ and $C$ such that, for every $N$, for every $z, w \in \Omega_{N}$, one has

$$
\left|\partial^{\alpha} g_{N}(z, w)\right| \leq C N^{-|\alpha| / 6}\left(1+|z|^{m}+|w|^{m}\right)\left|g_{N}(z, w)\right| .
$$

Recall now from Proposition A.3 that

$$
\left|g_{1}(z, w)\right| \leq e^{\frac{1}{4}|z-w|^{2}} .
$$

From the definition of $g_{N}$ one deduces that

$$
\left|g_{N}(z, w)\right| \leq e^{\frac{1}{4}|z-w|^{2}} .
$$

Thus the Taylor expansion of $g_{N}$ of order $6 K+5$ at the origin takes the following form:

$$
g_{N}(z, w)=\sum_{j=0}^{2 K+1} N^{-j / 2} b_{j}^{\psi}(z, w)+N^{-K-1} r_{K}^{\psi}(z, w, N) .
$$

Here, the $b_{j}^{\psi}$ are polynomials, and there exist $C$ and $m$ such that, for every $z, w$ and every $N$, one has

$$
\left|r_{K}^{\psi}(z, w, N)\right| \leq\left(1+|z|^{m}+|w|^{m}\right) e^{-\frac{1}{4}|z-w|^{2}} .
$$

We now return to equation (11). Replacing $A$ with $\pi^{n} \Pi_{1} e^{R_{A}}$, using the previous expression of $g_{N}$ and expanding, we find equation (9) with the desired control of $R_{K}$.

\section{References}

[1] V. Bargmann. On a Hilbert space of analytic functions and an associated integral transform. Part I. Comm. Pure. Appl. Math., 14(3):187$214,1961$.

[2] V. Bargmann. On a Hilbert space of analytic functions and an associated integral transform. Part II. Comm. Pure. Appl. Math., 20(1):1$101,1967$.

[3] F. A. Berezin. General concept of quantization. Comm. Math. Phys., 40:153-174, 1975. 
[4] R. Berman, B. Berndtsson, and J. Sjöstrand. A direct approach to Bergman kernel asymptotics for positive line bundles. Arkiv för Matematik, 46(2):197-217, 2008.

[5] D. Borthwick. Introduction to Kähler quantization. Contemporary Mathematics, 260:91, 2000.

[6] L. Boutet de Monvel. Intégration des équations de Cauchy-Riemann induites formelles. Séminaire Équations aux dérivées partielles (dit "Goulaouic-Schwartz"), pages 1-13, 1974-1975.

[7] L. Boutet de Monvel and V. Guillemin. The spectral theory of Toeplitz operators. Number 99 in Annals of Mathematics Studies. Princeton University Press, 1981.

[8] L. Boutet de Monvel and J. Sjöstrand. Sur la singularité des noyaux de Bergman et de Szegö. Journées équations aux dérivées partielles, 34-35:123-164, 1975.

[9] L. Charles. Aspects semi-classiques de la quantification géométrique. PhD thesis, Université Paris 9, 2000.

[10] L. Charles. Berezin-Toeplitz operators, a semi-classical approach. Communications in mathematical physics, 239(1-2):1-28, 2003.

[11] L. Charles and L. Polterovich. Sharp correspondence principle and quantum measurements. arXiv preprint arXiv:1510.02450, 2015.

[12] M. Christ. Upper bounds for Bergman kernels associated to positive line bundles with smooth hermitian metrics. arXiv preprint arXiv:1308.0062, 2013.

[13] X. Dai, K. Liu, and X. Ma. On the asymptotic expansion of Bergman kernels. J. differential Geometry, 72:1-41, 2006.

[14] B. Douçot and P. Simon. A semiclassical analysis of order from disorder. Journal of Physics A: Mathematical and General, 31(28):5855, 1998.

[15] G. Folland. Harmonic Analysis in phase space. Number 122 in Annals of Math. Studies. Princeton University Press, 1989.

[16] B. Helffer and D. Robert. Puits de potentiel généralisés et asymptotique semi-classique. In Annales de l'IHP Physique théorique, volume 41, pages 291-331, 1984. 
[17] B. Helffer and J. Sjöstrand. Multiple wells in the semi-classical limit I. Communications in Partial Differential Equations, 9(4):337-408, 1984.

[18] B. Helffer and J. Sjöstrand. Multiple wells in the semi-classical limit III : Interaction through non-resonant wells. Mathematische Nachrichten, 124(1):263-313, 1985.

[19] B. Helffer and J. Sjöstrand. Puits multiples en limite semi-classique II : Interaction moléculaire. symétries, perturbation. In Annales de l'IHP Physique théorique, volume 42, pages 127-212, 1985.

[20] B. Helffer and J. Sjöstrand. Puits multiples en limite semi-classique V : Étude des minipuits. Current topics in partial differential equations, pages 133-186, 1986.

[21] B. Helffer and J. Sjöstrand. Puits multiples en mécanique semiclassique VI : Cas des puits sous-variétés. In Annales de l'IHP Physique théorique, volume 46, pages 353-372, 1987.

[22] L. Hörmander. The analysis of linear partial differential operators. I. Distribution theory and Fourier analysis. Reprint of the second (1990) edition. Springer, Berlin, 2003.

[23] J. J. Kohn. Harmonic Integrals on Strongly Pseudo-Convex Manifolds: 1. Annals of Mathematics, pages 112-148, 1963.

[24] J. J. Kohn. Harmonic Integrals on Strongly Pseudo-Convex Manifolds: 2. Annals of Mathematics, pages 450-472, 1964.

[25] J. J. Kohn and H. Rossi. On the extension of holomorphic functions from the boundary of a complex manifold. Annals of Mathematics, pages 451-472, 1965.

[26] B. Kostant. Quantization and unitary representations. In Lectures in modern analysis and applications III, pages 87-208. Springer, 1970.

[27] Y. Le Floch. Singular Bohr-Sommerfeld conditions for 1D Toeplitz operators: elliptic case. Communications in Partial Differential Equations, 39(2):213-243, 2014.

[28] X. Ma and G. Marinescu. Holomorphic Morse inequalities and Bergman kernels, volume 254. Springer Science \& Business Media, 2007. 
[29] A. Melin. Lower bounds for pseudo-differential operators. Arkiv för Matematik, 9(1):117-140, 1971.

[30] S. Nakamura. Agmon-type exponential decay estimates for pseudodifferential operators. Journal of Mathematical Sciences, 5(4):693-712, 1998.

[31] N. Raymond and S. Vũ Ngọc. Geometry and spectrum in 2D magnetic wells. Annales de l'Institut Fourier, 65:137-169, 2015.

[32] M. Schlichenmaier. Deformation quantization of compact Kähler manifolds by Berezin-Toeplitz quantization. In Conférence Moshé Flato 1999, pages 289-306. Springer, 2000.

[33] B. Shiffman and S. Zelditch. Asymptotics of almost holomorphic sections of ample line bundles on symplectic manifolds. J. reine angew. Math., 544:181-222, 2002.

[34] B. Simon. Semiclassical analysis of low lying eigenvalues. I. Nondegenerate minima: Asymptotic expansions. In Annales de l'IHP Physique théorique, volume 38, pages 295-308, 1983.

[35] B. Simon. Semiclassical analysis of low lying eigenvalues. II. Tunnelling. In Ann. of Math., volume 120, pages 89-118, 1984.

[36] J.-M. Souriau. Quantification géométrique. applications. In Annales de l'institut Henri Poincaré (A) (N.S.), volume 6, pages 311-341. Gauthier-Villars, 1967.

[37] S. Vũ Ngọc. Formes normales semi-classiques des systèmes complètement intégrables au voisinage d'un point critique de l'application moment. Asymptotic Analysis, 24(3, 4):319-342, 2000.

[38] S. Vũ Ngọc. Bohr-Sommerfeld conditions for integrable systems with critical manifolds of focus-focus type. Communications on Pure and Applied Mathematics, 53(2):143-217, 2001.

[39] J. Williamson. On the algebraic problem concerning the normal forms of linear dynamical systems. American journal of mathematics, 58(1):141-163, 1936.

[40] N. M. J. Woodhouse. Geometric quantization. Oxford University Press, 1997.

[41] S. Zelditch. Szegö kernels and a theorem of Tian. Internat. Math. Res. Notices, 6:317-331, 1998. 